\title{
An addendum to the Heisenberg-Euler effective action beyond one loop
}

\author{
Holger Gies and Felix Karbstein \\ Helmholtz-Institut Jena, \\ Fröbelstieg 3, 07743 Jena, Germany \\ Theoretisch-Physikalisches Institut, Abbe Center of Photonics, \\ Friedrich-Schiller-Universität Jena, Max-Wien-Platz 1, 07743 Jena, Germany \\ E-mail: holger.gies@uni-jena.de, felix.karbstein@uni-jena.de
}

ABSTRACT: We study the effective interactions of external electromagnetic fields induced by fluctuations of virtual particles in the vacuum of quantum electrodynamics. Our main focus is on these interactions at two-loop order. We discuss in detail the emergence of the renowned Heisenberg-Euler effective action from the underlying microscopic theory of quantum electrodynamics, emphasizing its distinction from a standard one-particle irreducible effective action. In our explicit calculations we limit ourselves to constant and slowly varying external fields, allowing us to adopt a locally constant field approximation. One of our main findings is that at two-loop order there is a finite one-particle reducible contribution to the Heisenberg-Euler effective action in constant fields, which was previously assumed to vanish. In addition to their conceptual significance, our results are relevant for high-precision probes of quantum vacuum nonlinearity in strong electromagnetic fields.

KEYWORDS: Effective field theories, Nonperturbative Effects

ArXiv EPrint: 1612.07251 


\section{Contents}

1 Introduction

2 1PI effective action and Heisenberg-Euler effective action - Differences and common ground $\quad 3$

2.1 Partition function and vacuum persistence amplitude in an external field 3

2.2 From QED to the Heisenberg-Euler effective action 5

2.3 Diagrammatic expansion of the Heisenberg-Euler effective action 8

3 The Heisenberg-Euler effective action in constant electromagnetic fields 11

4 Effective theory of low-frequency photons in slowly varying electromagnetic fields

$\begin{array}{lll}4.1 & \text { Locally constant field approximation } & 13\end{array}$

$\begin{array}{lll}4.2 & \text { Effective action for low-frequency photons } & 14\end{array}$

$\begin{array}{lll}4.3 & \text { 1PR contribution to the Heisenberg-Euler effective action } & 16\end{array}$

$\begin{array}{lll}4.4 & \text { Low-frequency photon propagation } & 17\end{array}$

5 Conclusions and outlook $\quad 23$

A Useful derivative identities $\quad 24$

B Strong-field asymptotics for spinor QED 25

B.1 One-loop Heisenberg-Euler effective Lagrangian in constant external fields 25

B.2 Two-loop effective Lagrangian in constant external fields 26

\section{Introduction}

One of the striking predictions of quantum field theory (QFT) is that virtual charged particle-antiparticle fluctuations in the quantum vacuum can induce nonlinear interactions among electromagnetic fields [1-3]; for reviews emphasizing various theoretical aspects as well as prospects for the experimental detection of such effects, see [4-13]. Aiming at probing the vacuum of the Standard Model of particle physics with classical electromagnetic fields and low energy photons, the dominant effective interactions are governed by quantum electrodynamics (QED).

For the macroscopic electromagnetic fields presently attainable in the laboratory, the effects of QED vacuum nonlinearities are rather small, making their experimental detection challenging $[9,11]$. These effective interactions have no tree-level analogue, but are mediated by at least one electron-positron loop. For electromagnetic fields which vary on scales much larger than the Compton wavelength of the electron $\lambda=3.86 \cdot 10^{-13} \mathrm{~m}$ and are weak in comparison to the critical electric field strength $E_{\mathrm{cr}} \equiv \frac{m^{2} c^{3}}{e \hbar} \simeq 1.3 \cdot 10^{18} \mathrm{~V} / \mathrm{m}[2,14,15]$, 
i.e., fulfill $\{|\vec{E}|, c|\vec{B}|\} \ll E_{\text {cr }}$, this results in a parametric suppression of the effective interaction by inverse powers of the electron mass. Most of the electromagnetic fields available in the laboratory meet this criterion.

The effective interactions can in particular impact photon propagation and give rise to remarkable effects such as vacuum birefringence experienced by probe photons traversing a classical electromagnetic field [16-19]; for ongoing experimental efforts aiming at the verification of vacuum birefringence using macroscopic fields, see [20-25]; for theoretical proposals advocating dedicated high-intensity laser experiments, see [26-35]. Recently, indications have been reported for the relevance of QED vacuum birefringence for optical polarimetry of a neutron star [36]. Other theoretical proposals for optical signatures of quantum vacuum nonlinearity have focused on photon-photon scattering in the form of laser-pulse collisions [37-40], interference effects [41-44], quantum reflection [45, 46], photon merging [47-51], photon splitting [19, 52-60], and higher-harmonic generation from laser driven vacuum [61-64]. Finally, and perhaps most strikingly, strong electric fields can facilitate the spontaneous formation of real electron-position pairs from the QED vacuum via the Schwinger effect $[2,14,15]$.

It is a fascinating aspect of this plethora of phenomena that they manifest the effective interactions of electromagnetic fields beyond Maxwell's linear theory, which can be summarized elegantly by an effective action that dates back to the early days of quantum field theory $[2,3]$ : the Heisenberg-Euler effective action. Its matured embedding into the modern language of field theory is due to Schwinger [15], who gave a nonperturbative definition of this action by means of the vacuum persistence amplitude, i.e., the Schwinger functional. Nowadays, QFT is often defined in terms of generating functionals for correlation functions, with the concept of the effective action being identified with the generating functions of one-particle irreducible (1PI) correlators (proper vertices).

In this article, we emphasize that the Heisenberg-Euler effective action is different from - though related to - the 1PI effective action. This fact has, of course, been well known in the specialized literature $[4,65]$ but is sometimes confused in textbooks. We detail the construction of the Heisenberg-Euler effective action from the standard definition of QED in terms of the partition function in the present work. The difference between the two effective actions is manifested by one-particle reducible (1PR) contributions to the Heisenberg-Euler action. In a perturbative loop expansion, such 1PR contributions occur at and beyond the two-loop order. At two-loop order, we find that there is a finite 1PR contribution to the Heisenberg-Euler effective action in constant electromagnetic fields, which was previously believed to vanish.

Using a locally constant field approximation (LCFA), we also study in detail the effective theory of slowly varying classical background fields and low-frequency photon fields in the QED vacuum. The photon polarization tensor derived within the latter contains 1PI, 1PR, as well as disconnected contributions, all of which can be understood as generated by the 1PI effective action.

As higher-loop diagrams are typically suppressed in comparison to the one-loop diagram, a proper inclusion of the previously neglected 1PR diagrams is expected to impact the proposed experimental signatures of quantum vacuum nonlinearities only at subleading 
order. In fact, most of the theoretical studies listed above exclusively limit themselves to one-loop order. For instance for vacuum birefringence in weak fields, the two-loop contribution represents only a $1 \%$ correction [66].

Our article is organized as follows. Section 2 is devoted to an in-depth discussion of the Heisenberg-Euler effective action. Here, we elaborate differences and common ground with respect to the standardized 1PI effective action and show how the Heisenberg-Euler effective action emerges from the microscopic theory of QED. Finally, we explicitly sketch its diagrammatic expansion up to two-loop order. In section 3 we focus on the Heisenberg-Euler effective action in constant fields. Here, we provide the weak- and strong-field asymptotics of the Heisenberg-Euler effective Lagrangian at one- and two-loop order. Their explicit derivation is relegated to appendix B. Thereafter, in section 4 we introduce the LCFA and show how it can be employed to construct an effective theory describing the interactions of slowly varying electromagnetic fields and low-frequency photon fields in the QED vacuum. Here, we mainly concentrate on fluctuation-induced effective interactions at two-loop order. Finally, we end with conclusions and an outlook in section 5.

\section{1PI effective action and Heisenberg-Euler effective action - Differ- ences and common ground}

\subsection{Partition function and vacuum persistence amplitude in an external field}

The Heisenberg-Euler action $\Gamma_{\mathrm{HE}}[2]$ is often viewed as the prototype of an effective action $\Gamma$, the latter having become a canonized central object in QFT. Effective actions $\Gamma$ have a precise meaning as the generating functional of 1PI correlators (proper vertices) and follow from a standardized QFT construction via the Legendre transform of the partition function ${ }^{1}$

$$
\begin{aligned}
Z[J] & =\int \mathcal{D} \varphi e^{i S[\varphi]+i \int J \varphi}, \\
\Rightarrow \quad \Gamma[\phi] & =\sup _{J}\left[-\int J \phi-i \ln Z[J]\right] .
\end{aligned}
$$

Here, $S$ denotes the classical action of the theory to be quantized, $\varphi$ summarizes the fluctuation fields such as electrons/positrons and photons in QED, and $J$ is a source that can be used to generate correlation functions from the partition function. The above definition (2.2) guarantees the 1PI property of $\Gamma$, making it a convenient and elegant tool for many purposes of QFT.

As it is of particular relevance for understanding our novel results obtained below, we wish to emphasize that the Heisenberg-Euler action does not fall into the class of effective actions as constructed from eq. (2.2). This statement holds both from the perspective of its historical construction as well as from its modern use in strong-field physics.

The physical difference is, for instance, apparent from the fact that the 1PI effective action (2.2) depends on the so-called classical field $\phi$. The supremum prescription in

\footnotetext{
${ }^{1}$ In order to keep the notation compact, we employ the shorthand forms $\int_{x} \equiv \int \mathrm{d}^{4} x$ and $\int_{k} \equiv \int \frac{\mathrm{d}^{4} k}{(2 \pi)^{4}}$ for the integrations over position and momentum space, respectively. Besides, we simply use $\int$ if the integration can be performed in position or momentum space.
} 
eq. (2.2) relates $\phi$ to the expectation value of the fluctuating quantum field,

$$
\phi=\frac{1}{i} \frac{1}{Z[J]} \frac{\delta Z[J]}{\delta J}=\langle\varphi\rangle,
$$

(a relation that can be considered both at $J=0$ or for a nonvanishing source). In turn, the field $\phi$ is obviously the result of a full quantum averaging process.

By contrast, Heisenberg and Euler [2] as well as Weisskopf [3] have been interested in the response of the quantized electron-positron field to a non-quantized external electromagnetic field $\bar{A}$ which is considered to be given from the outside. In absence of quantum fluctuations, this external field would obey an action principle with action $S_{\text {ext }}[\bar{A}]=$ $-\frac{1}{4} \int \bar{F}_{\mu \nu} \bar{F}^{\mu \nu}$, where $\bar{F}_{\mu \nu}=\partial_{\mu} \bar{A}_{\nu}-\partial_{\nu} \bar{A}_{\mu}$. Contrarily, in the presence of quantum fluctuations, the dynamics of the external field $\bar{A}$ is governed by the Heisenberg-Euler action,

$$
\Gamma_{\mathrm{HE}}[\bar{A}]=S_{\mathrm{ext}}[\bar{A}]+W[\bar{A}]
$$

The additional contribution $W[\bar{A}]$ arising from quantum fluctuations has been formalized by Schwinger in terms of the vacuum persistence amplitude [15],

$$
\left\langle 0_{+} \mid 0_{-}\right\rangle^{\bar{A}}=e^{i W[\bar{A}]},
$$

parametrizing the probability amplitude for the vacuum to persist in the presence of an external field $\bar{A}$ ("the prescribed field" $[15]$ ). The Schwinger functional $W[\bar{A}]$ is considered to be a functional of the external field (and not of a source coupled to a quantum field). It can be written as a path integral over fluctuating fields,

$$
e^{i W[\bar{A}]}=\int \mathcal{D} q e^{i \int\left(-\frac{1}{4} Q_{\mu \nu} Q^{\mu \nu}\right)} e^{i S_{\psi}[\bar{A}+q]},
$$

where we employed the shorthand notation

$$
e^{i S_{\psi}[\bar{A}+q]}=\int \mathcal{D} \bar{\psi} \mathcal{D} \psi e^{i \int \bar{\psi}(-i \not D[\bar{A}+q]+m) \psi} .
$$

For a proper comparison with the literature, we point out that our phase conventions agree with those of [4] and thus do not include the Maxwell term for the $\bar{A}$ field in $W[\bar{A}]$ (contrary to Schwinger's conventions [15]). ${ }^{2}$

In eq. (2.6), we have distinguished between the external background $\bar{A}$ and the fluctuating photon field $q$, the latter being equipped with a kinetic term involving the field strength $Q_{\mu \nu}=\partial_{\mu} q_{\nu}-\partial_{\nu} q_{\mu}$. The external field couples to the fermions $\psi, \bar{\psi}$, and hence the result of the path integral depends parametrically on $\bar{A}$.

We emphasize that eq. (2.6) contains no information about the dynamics that creates $\bar{A}$ in the first place. This has to be provided by a separate theory for the external field, which is conventionally assumed to obey an action principle with action $S_{\text {ext }}[\bar{A}]$. In absence

\footnotetext{
${ }^{2}$ More precisely, our conventions agree with those of the defining equations (1.45) and (1.48) of [4]; these are slightly different from those of chapter 7 of $[4]$ where $W[\bar{A}]$ denotes the electron-positron loop.
} 
of quantum fluctuations, $\bar{A}$ would be a solution of this external theory and its equations of motion given by

$$
\frac{\delta S_{\mathrm{ext}}[\bar{A}]}{\delta \bar{A}_{\mu}}=-\bar{J}^{\mu},
$$

where $\bar{J}$ is a classical source for the external field. Upon the inclusion of quantum fluctuations, the dynamics of $\bar{A}$ is modified such that $\Gamma_{\mathrm{HE}}[\bar{A}]$ governs the dynamics of the external field.

To one-loop order, the photon fluctuations $\sim \mathcal{D} q$ can be ignored in eq. (2.6) and one obtains the historic answer $[2,3,15]$. At higher loops, starting from two loop on, $W[\bar{A}]$ also contains one-particle reducible diagrams [4, 65, 67], as is obvious from its definition (2.6) and will be recalled explicitly below. Hence, $\Gamma_{\mathrm{HE}}$ does not correspond to the standard 1PI effective action.

From a fundamental viewpoint, the concept of a non-quantized external field $\bar{A}$ seems somewhat redundant, as the world is fully quantum. Moreover, a separation into internal and external fields might seem purely academic. Nevertheless, this concept is perfectly adjusted to our perception of a real experiment in terms of classically controlled sources and detectors. In the remainder of this section, we detail how this useful concept can be extracted from the full quantum theory.

\subsection{From QED to the Heisenberg-Euler effective action}

In order to develop the formalism, it is useful to envisage a typical physical system where the external field $\bar{A}$ is generated by suitable sources $\bar{J}$. The sources (lasers, magnets, etc.) are macroscopically separated from an interaction region of volume $V_{\mathrm{I}}$ (focal volume, interaction cavity, etc.). We consider physical situations where quantum vacuum nonlinearities, i.e., higher-order effective couplings of electromagnetic fields mediated by quantum fluctuations of charged particles, become sizable only within $V_{\mathrm{I}}$.

Then, the physics inside $V_{\mathrm{I}}$ can create signals (induced field components, signal photons, etc.) which are ultimately observed in detectors macroscopically separated from the interaction region $V_{\mathrm{I}}$. Due to the smallness of the nonlinear effective couplings among electromagnetic fields induced by quantum fluctuations of virtual charged particles, the signal may often be of quantum nature, as it is, e.g., the case for a single-photon signal to be measured in a single-photon detector. Still, it is useful to think of the signal as a contribution to the external field $\bar{A}$, because it is ultimately measured far away from the region $V_{\mathrm{I}}$.

In order to distinguish between applied fields $\bar{A}_{\text {applied }}$ (e.g., the fields provided by lasers, or magnets) and the signal photons $\bar{A}_{\text {signal }}$, one may decompose the external field as

$$
\bar{A}=\bar{A}_{\text {applied }}+\bar{A}_{\text {signal }} \text {. }
$$

As the signal $\bar{A}_{\text {signal }}$ is eventually induced by $\bar{A}_{\text {applied }}$, the two components of $\bar{A}$ will typically exhibit a causal ordering in time. Similarly, it is possible to distinguish between the source parts $\bar{J}$ that are responsible for creating $\bar{A}_{\text {applied }}$ and those that interact with $\bar{A}_{\text {signal }}$ within the detectors. 
Now, the quantitative success of classical electrodynamics heuristically implies that the effective self-interactions as well as mutual couplings of $\bar{A}_{\text {signal }}$ and $\bar{A}_{\text {applied }}$ mediated by quantum fluctuations $(\psi, \bar{\psi}, q)$ are dramatically suppressed and essentially vanish outside the interaction volume $V_{\mathrm{I}}$. From the viewpoint of QED, this is a consequence of the locality of the theory and the smallness of its coupling. This establishes an operational definition of $\bar{A}$ in the outside region, where it is related to the sources $\bar{J}$ which control both the creation of $\bar{A}_{\text {applied }}$ and the detection of $\bar{A}_{\text {signal }}$. For the following formalism, it suffices to just refer to the combined field $\bar{A}$. From a conceptual point of view, the details of the choice of $V_{\mathrm{I}}$ do not really matter. It is the possibility of a partitioning of the system into an internal interaction and external Maxwellian region that matters (cf. below). Correspondingly, there is no need to consider (Casimir-like) effects due to the finite volume of $V_{\mathrm{I}}$ : the interaction volume can always be chosen large enough to render such effects negligible. In fact, as not even a physical boundary is necessary, the transition between internal and external regions can be fuzzy.

Apart from the effects of quantum corrections, we expect $\bar{A}$ to satisfy a classical Maxwell equation $\delta S_{\text {ext }} / \delta \bar{A} \simeq-\bar{J}$. More precisely, we assume $\bar{A}$ to be defined as the solution of the following equation,

$$
\partial_{\mu} \bar{F}^{\mu \nu}+C^{\nu}[\bar{A}]=-\bar{J}^{\nu}
$$

where $C^{\nu}[\bar{A}]$ parameterizes quantum corrections which should be negligible in the outside region, i.e., approximately fulfill $C^{\nu}[\bar{A}]=0$ outside $V_{\mathrm{I}}$. By contrast, $C^{\nu}[\bar{A}]$ can become relevant in the interaction region $V_{\mathrm{I}}$, where however $\bar{J}=0$. In QED, $C^{\nu}[\bar{A}]$ is perturbatively of $\mathcal{O}(\alpha)$ and nonlinear and nonlocal in the field, with the nonlinearities and nonlocalities being controlled by the Compton scale.

With these prerequisites, let us turn to the standard partition function for QED,

$$
Z[J]=\int \mathcal{D} A e^{i \int\left[-\frac{1}{4} F_{\mu \nu} F^{\mu \nu}+J_{\mu} A^{\mu}\right]} e^{i S_{\psi}[A]} .
$$

Concentrating on correlation functions of the electromagnetic field, we only include a source term for the gauge field. Of course, the generalization to sources for the fermions is straightforward. In a next step, we employ the variable substitution

$$
A=\bar{A}+q,
$$

in order to rewrite eq. (2.11) as

$$
Z[J]=e^{i \int\left[-\frac{1}{4} \bar{F}_{\mu \nu} \bar{F}^{\mu \nu}+J_{\mu} \bar{A}^{\mu}\right]} \int \mathcal{D} q e^{i \int\left[-\frac{1}{4} Q_{\mu \nu} Q^{\mu \nu}+\left(\partial_{\mu} \bar{F}^{\mu \nu}+J^{\nu}\right) q_{\nu}\right]} e^{i S_{\psi}[\bar{A}+q]}
$$

We emphasize that - despite its explicit appearance on the right-hand side - this partition function of course does not depend on $\bar{A}$ but is a functional of the source $J$ only. Contrary to the standard QFT treatment where $J$ often plays the role of an auxiliary variable, the source is needed here to sustain the external field. Still, let us not simply reduce $J \rightarrow \bar{J}$, but keep it slightly more general. 
As a next step, we classicalize the external field $\bar{A}$ : for this, we assume that the fluctuation field $q$ only couples to the electron-positron field, i.e., any direct coupling to the background field should vanish. More precisely, we choose $J$ such that

$$
\int d^{4} x\left(\partial_{\mu} \bar{F}^{\mu \nu}+J^{\nu}\right) q_{\nu}=0 \quad \leftrightarrow \quad J^{\nu}=-\partial_{\mu} \bar{F}^{\mu \nu}=:-(\partial \bar{F})^{\nu} .
$$

Let us emphasize that for any violation of eq. (2.14), i.e., $\partial_{\mu} \bar{F}^{\mu \nu}+J^{\nu}=\mathcal{J}^{\nu} \neq 0$, the remnant source $\mathcal{J}^{\nu}$ could potentially induce a nonvanishing expectation value $\left\langle q_{\nu}\right\rangle=$ $(1 / i Z)\left(\delta Z / \delta \mathcal{J}^{\nu}\right) \neq 0$. Such an expectation value could mix with $\bar{A}$ and thereby lead to inconsistencies with the concept of $\bar{A}$ being an external field.

For sources fulfilling eq. (2.14), we have

$$
\left.Z[J]\right|_{J=-(\partial \bar{F})}=\mathrm{e}^{i \int\left(+\frac{1}{4} \bar{F}_{\mu \nu} \bar{F}^{\mu \nu}\right)} \int \mathcal{D} q e^{i \int\left(-\frac{1}{4} Q_{\mu \nu} Q^{\mu \nu}\right)} e^{i S_{\psi}[\bar{A}+q]} .
$$

A comparison with Schwinger's vacuum persistence amplitude (2.6) shows that

$$
\left.Z[J]\right|_{J=-(\partial \bar{F})}=\mathrm{e}^{i \int\left(+\frac{1}{4} \bar{F}_{\mu \nu} \bar{F}^{\mu \nu}\right)} e^{i W[\bar{A}]} .
$$

This suggests introducing the Heisenberg-Euler action by

$$
\begin{aligned}
\Gamma_{\mathrm{HE}}[\bar{A}] & :=\left.\left[-\int J_{\mu} \bar{A}^{\mu}-i \ln Z[J]\right]\right|_{J=-(\partial \bar{F})} \\
& =-\int \frac{1}{4} \bar{F}_{\mu \nu} \bar{F}^{\mu \nu}+W[\bar{A}] .
\end{aligned}
$$

Note that eq. (2.17) does not constitute a Legendre transform, since $J$ is subject to the constraint (2.14).

Since the field $\bar{A}$ is ultimately created by the classical source $\bar{J}$, we demand for

$$
-\bar{J}^{\mu} \stackrel{!}{=} \frac{\delta \Gamma_{\mathrm{HE}}[\bar{A}]}{\delta \bar{A}_{\mu}}=\partial_{\nu} \bar{F}^{\nu \mu}+\frac{\delta W[\bar{A}]}{\delta \bar{A}_{\mu}},
$$

which implies that the correction term in eq. (2.10) is given by $C^{\mu}[\bar{A}]=\delta W[\bar{A}] / \delta \bar{A}_{\mu}$. Hence the correction term can be viewed as a shift in the source term

$$
\bar{J} \rightarrow \bar{J}+C[\bar{A}]=J,
$$

which is needed in eq. (2.14) to inhibit that the background as well as $\bar{J}$ provide a source for the fluctuation field $q$. If we had defined the field $\bar{F}$ in terms of the source $\bar{J}$ in combination with the classical field equation $\partial_{\mu} \bar{F}^{\mu \nu}=-\bar{J}^{\nu}$, we would have arrived at the same definition (2.17) for the Heisenberg-Euler action. However, this definition of the external field would have been inconsistent with the quantum equation of motion (2.18) from order $\alpha$ on.

This concludes our derivation of the Heisenberg-Euler effective action $\Gamma_{\mathrm{HE}}$ from the standard QFT partition function of QED. The result (2.17) is in perfect agreement with Schwinger's definition by means of the vacuum persistence amplitude (2.5). Our derivation 


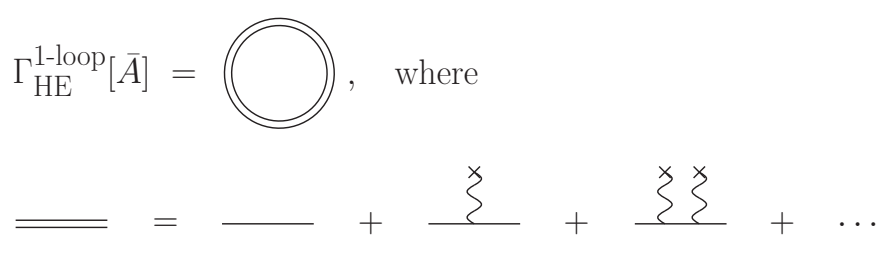

Figure 1. Diagrammatic representation of the one-loop Heisenberg-Euler effective action. The double line denotes the dressed fermion propagator accounting for arbitrarily many couplings to the external field $\bar{A}$, represented by the wiggly lines ending at crosses.

underpins once more that $\Gamma_{\mathrm{HE}}$ is decisively different from the standard effective action $\Gamma$, as it also contains one-particle reducible contributions which contribute to the equations of motion of the external field.

We end this section with the remark that once $\Gamma_{\mathrm{HE}}$ is obtained, it can be used for determining $\bar{A}$ both by purely classical means or by describing $\bar{A}$ in terms of a Fock space in a quantum optical setting. Both treatments of $\bar{A}$ are useful as well as legitimate. In particular, it is natural to treat applied macroscopic fields $\bar{A}_{\text {applied }}$ classically and the induced weak signal fields $\bar{A}_{\text {signal }}$ by means of Fock space states, as has been suggested in the vacuum emission picture [63].

\subsection{Diagrammatic expansion of the Heisenberg-Euler effective action}

Apart from the classical Maxwell term, the Heisenberg-Euler effective action (2.17) is given by the Schwinger functional $W[\bar{A}]$, which can be defined in terms of a functional integral, see eq. (2.6). The latter encodes quantum corrections giving rise to effective self-interactions of the external electromagnetic field; for $\hbar \rightarrow 0$ we have $W[\bar{A}] \rightarrow 0$. It can be perturbatively expanded by standard techniques, cf., e.g., [4]. Generically, this expansion can be organized in the number of loops,

$$
W[\bar{A}]=\sum_{l=1}^{\infty} \Gamma_{\mathrm{HE}}^{l \text {-loo }}[\bar{A}],
$$

with $\Gamma_{\mathrm{HE}}^{l \text {-loop }} \sim\left(\frac{\alpha}{\pi}\right)^{l-1}$, where $\alpha=\frac{e^{2}}{4 \pi} \simeq \frac{1}{137}$ is the fine-structure constant; we use the Heaviside-Lorentz System with $c=\hbar=1$. At each loop order $l, \Gamma_{\mathrm{HE}}^{l \text {-loop }}=\int_{x} \mathcal{L}_{\mathrm{HE}}^{l \text {-loop }}$ accounts for an infinite number of couplings to the external field, and thus is fully nonperturbative in the parameter $e \bar{A}$. For completeness, we sketch the expansion to two-loop order in the following. We begin by noting that the fermionic integral in eq. (2.7) can be written as a functional determinant,

$$
i S_{\psi}[\bar{A}+q]=\ln \operatorname{det}(-i \not D[\bar{A}+q]+m) .
$$

If evaluated at $q=0$, this quantity amounts to the one-loop Heisenberg-Euler effective action in the external field $\bar{A}$, i.e., $\Gamma_{\mathrm{HE}}^{1-\text { loop }}[\bar{A}]=S_{\psi}[\bar{A}]$; for a graphical representation, cf. figure 1. Since $S_{\psi}$ is a one-loop expression, the two-loop order of the Schwinger functional is already obtained by performing the photonic fluctuation integral $\sim \mathcal{D} q$ to Gaußian order. 
For this, we expand $S_{\psi}$ about the external field $\bar{A}$,

$$
S_{\psi}[\bar{A}+q]=S_{\psi}[\bar{A}]+\int\left(S_{\psi}^{(1)}[\bar{A}]\right)^{\mu} q_{\mu}+\frac{1}{2} \iint q_{\mu}\left(S_{\psi}^{(2)}[\bar{A}]\right)^{\mu \nu} q_{\nu}+\mathcal{O}\left(q^{3}\right),
$$

where we employed the shorthand notation

$$
\left(S_{\psi}^{(n)}\right)^{\sigma_{1} \ldots \sigma_{n}}[\bar{A}]:=\left.\frac{\delta^{n} S_{\psi}[A]}{\delta A_{\sigma_{1}} \ldots \delta A_{\sigma_{n}}}\right|_{A=\bar{A}} .
$$

The first-order term corresponds to a one-loop photon current induced by the field $\bar{A}$, and the Hessian is related to the one-loop photon polarization tensor $\Pi^{\mu \nu}[\bar{A}]:=\left(S_{\psi}^{(2)}\right)^{\mu \nu}[\bar{A}]$ evaluated in the external field $\bar{A}$; for completeness note that this definition of the photon polarization tensor differs from that of [68] by an overall minus sign. To Gaußian order, we ignore the terms of $\mathcal{O}\left(q^{3}\right)$ in the exponent, resulting in

$$
e^{i W[\bar{A}]} \simeq e^{i S_{\psi}[\bar{A}]} \int \mathcal{D} q e^{i \int\left(S_{\psi}^{(1)}[\bar{A}]\right)^{\mu} q_{\mu}-\frac{i}{2} \iint q_{\mu}\left(D^{-1}-\Pi[\bar{A}]\right)^{\mu \nu} q_{\nu}}
$$

In principle, terms of $\mathcal{O}\left(q^{3}\right)$ in the exponent can, of course, be treated perturbatively to any desired order. The quantity $\left(D^{-1}\right)^{\mu \nu}$ arises from the Maxwell term for the fluctuations and denotes the inverse photon propagator. E.g., in momentum space and accounting for a gauge-fixing term (generalized Lorenz gauge), we have

$$
D^{\mu \nu}(p)=\frac{1}{p^{2}-i \epsilon}\left(g^{\mu \nu}-(1-\xi) \frac{p^{\mu} p^{\nu}}{p^{2}-i \epsilon}\right)
$$

where $\xi=1$ in the Feynman gauge. Performing the integration over $q$ in eq. (2.24), we arrive at

$$
e^{i W[\bar{A}]} \simeq e^{i S_{\psi}[\bar{A}]} e^{\frac{i}{2} \iint\left(S_{\psi}^{(1)}[\bar{A}]\right)_{\mu}\left[\left(D^{-1}-\Pi[\bar{A}]\right)^{-1}\right]^{\mu \nu}\left(S_{\psi}^{(1)}[\bar{A}]\right)_{\nu} \operatorname{det}^{-1 / 2}\left(D^{-1}-\Pi[\bar{A}]\right) .}
$$

To Gaußian order in the photon fluctuations, we thus obtain for the Schwinger functional

$$
W[\bar{A}] \simeq S_{\psi}[\bar{A}]-\frac{1}{2} \ln \operatorname{det}\left(D^{-1}-\Pi[\bar{A}]\right)+\frac{1}{2} \iint\left(S_{\psi}^{(1)}[\bar{A}]\right)_{\mu}\left[\left(D^{-1}-\Pi[\bar{A}]\right)^{-1}\right]^{\mu \nu}\left(S_{\psi}^{(1)}[\bar{A}]\right)_{\nu} .
$$

The first term on the right-hand side corresponds to the one-loop contribution to the Heisenberg-Euler effective action (called $W^{(1)}[\bar{A}]$ in $[4,15]$ ). The other two terms contain the complete two-loop order contribution as well as subclasses of diagrams to arbitrarily high loop order. To make this manifest, we expand the ln det term as follows,

$$
\begin{aligned}
\ln \operatorname{det}\left(D^{-1}-\Pi[\bar{A}]\right) & =\operatorname{Tr} \ln (1-D \Pi[\bar{A}])+\operatorname{Tr} \ln D^{-1} \\
& =-\operatorname{Tr}(D \Pi)+\frac{1}{2} \operatorname{Tr}(D \Pi D \Pi)+\mathcal{O}\left(\Pi^{3}\right),
\end{aligned}
$$

where in the last step, we have dropped field-independent constants. The $\operatorname{Tr}(D \Pi)$ term corresponds exactly to the two-loop contribution to the Heisenberg-Euler action that has first been computed in [65]; see also [4, 69-71]. This contribution as well as all higher order terms in eq. (2.28) are one-particle irreducible from a diagrammatic viewpoint; see figure 2. 


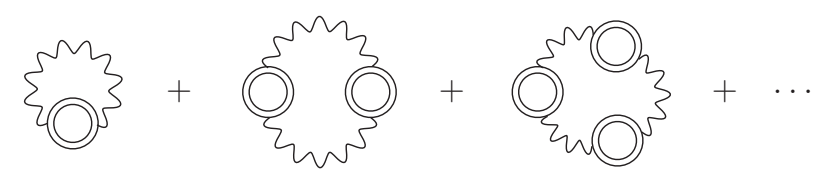

Figure 2. Diagrammic representation of eq. (2.28). The wiggly line is the photon propagator; for the definition of the double line, cf. figure 1.

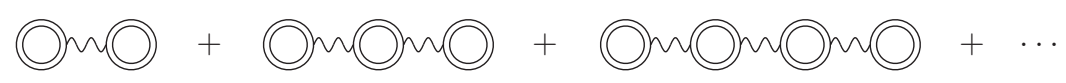

Figure 3. One-particle reducible diagrams constituting the last term in eq. (2.26). For the definition of the double line, cf. figure 1.

$$
\Gamma_{\mathrm{HE}}^{2-\operatorname{loop}}[\bar{A}]=(m y+\infty
$$

Figure 4. Diagrams constituting the two-loop Heisenberg-Euler effective action. Obviously, we have $\Gamma_{\mathrm{HE}}^{2 \text {-loop }}=\left.\Gamma_{\mathrm{HE}}^{2 \text {-loop }}\right|_{1 \mathrm{PI}}+\left.\Gamma_{\mathrm{HE}}^{2 \text {-loop }}\right|_{1 \mathrm{PR}}$. Note that the first diagram amounts to the leftmost one in figure 2, where it is drawn in a slightly different way; for the definition of the double line, cf. figure 1 .

The last term in eq. (2.26), however, contains

$$
\left(D^{-1}-\Pi[\bar{A}]\right)^{-1}=D-D \Pi D+D \Pi D \Pi D+\ldots,
$$

corresponding to the Dyson series of the full one-loop resummed photon propagator. In the last term of eq. (2.26), this resummed propagator interconnects two one-loop photon currents $\sim\left(S_{\psi}^{(1)}[\bar{A}]\right)$. All the diagrams arising when adopting the expansion (2.29) in the last term in eq. (2.26) are one-particle reducible; see figure 3.

In turn, the two-loop Heisenberg-Euler effective action consists of a 1PI and a 1PR diagram and is given by

$$
\Gamma_{\mathrm{HE}}^{2 \text {-loop }}[\bar{A}]=\underbrace{\frac{1}{2} \operatorname{Tr}(D \Pi[\bar{A}])}_{=:\left.\Gamma_{\mathrm{HE}}^{2-\text {-loo }}\right|_{1 \mathrm{PI}}}+\underbrace{\frac{1}{2} \iint\left(S_{\psi}^{(1)}[\bar{A}]\right)_{\mu} D^{\mu \nu}\left(S_{\psi}^{(1)}[\bar{A}]\right)_{\nu}}_{=:\left.\Gamma_{\mathrm{HE}}^{2 \text {-loop }}\right|_{1 \mathrm{PR}}} .
$$

The existence as a matter of principle of the 1PR term in eq. (2.30) has been known for a long time. It has, however, been argued that this term vanishes for constant external fields $[4,65]$. Let us reproduce this argument for reasons of completeness: a crucial building block of the $1 \mathrm{PR}$ term is $\left(S_{\psi}^{(1)}[\bar{A}]\right)_{\nu}$, which corresponds to the one-loop photon current which will be called $j_{1 \text {-loop }}^{\mu}[\bar{A}]$ below. For a constant external field, $j_{1 \text {-loop }}^{\mu}[\bar{A}]$ does not depend on any spacetime point $x$ either. On the other hand, $j_{1 \text {-loop }}^{\mu}[\bar{A}]$ is a Lorentz 4vector. The vector index of the current can only be generated from the building blocks $\bar{F}$, $\partial$ and $x$. However, for constant fields $\partial^{\mu} F^{\nu \kappa}=0$ and for an $x$ independent current, all conceivable combinations with one vector index vanish and so does the current (an explicit verification of this fact in momentum space is given below). 
While this part of the argument holds true in the full analysis, it does not necessarily imply that the 1PR diagram in figure 4 vanishes. In fact, the two currents in the 1PR diagram are convoluted with a photon propagator, describing a long-range force with an IR singularity $\sim 1 / p^{2}$ in the propagator. Hence, it is a quantitative question as to whether the currents approaching zero are outbalanced by the IR singularity of the photon propagator. In the subsequent sections, we give proof that the result is finite.

Heuristically, it is clear that the above-mentioned argument for the vanishing of the current no longer holds as soon as the external field supports a slightest inhomogeneity somewhere in spacetime. So, $j^{\mu}[\bar{A}]=0$ strictly relies on $\partial^{\mu} F^{\nu \kappa}=0$ for all $x$. On the other hand, the existence of massless long-range fluctuations in QED is independent of the constant-field assumption. Therefore, the $1 \mathrm{PR}$ term is expected to be finite for any realistic field.

\section{The Heisenberg-Euler effective action in constant electromagnetic fields}

In the following, we first summarize some of our explicit results for the Heisenberg-Euler effective action, concentrating on fully analytic expressions in asymptotic field-strength limits for spinor QED. This provides for a first glance at the parametric dependence of the various contributions, and elucidates the regime of relevance of the two-loop 1PR term. Details of the calculations are deferred to the subsequent sections.

For constant external electromagnetic fields, $\bar{F}^{\mu \nu}=$ const., Lorentz and gauge invariance constrain $\Gamma_{\mathrm{HE}}$ to depend on $\bar{A}$ only in terms of the two scalar invariants $[1,2]$ $\mathcal{F}=\frac{1}{4} \bar{F}_{\mu \nu} \bar{F}^{\mu \nu}=\frac{1}{2}\left(\vec{B}^{2}-\vec{E}^{2}\right)$ and $\mathcal{G}=\frac{1}{4} \bar{F}_{\mu \nu}{ }^{*} \bar{F}^{\mu \nu}=-\vec{B} \cdot \vec{E}$, with dual field strength tensor ${ }^{*} \bar{F}^{\mu \nu}=\frac{1}{2} \epsilon^{\mu \nu \alpha \beta} \bar{F}_{\alpha \beta}$. Here, $\epsilon^{\mu \nu \alpha \beta}$ is the totally antisymmetric tensor $\left(\epsilon^{0123}=1\right)$, and our metric convention is $g_{\mu \nu}=\operatorname{diag}(-1,+1,+1,+1)$. In addition, CP invariance of QED dictates $\Gamma_{\mathrm{HE}}$ to be even in the pseudoscalar quantity $\mathcal{G}, \Gamma_{\mathrm{HE}}[\bar{A}]=\Gamma_{\mathrm{HE}}\left(\mathcal{F}, \mathcal{G}^{2}\right)$. To keep notations compact, we also introduce the dimensionless quantities $\tilde{\mathcal{F}}=\left(\frac{e}{m^{2}}\right)^{2} \mathcal{F}$ and $\tilde{\mathcal{G}}=\left(\frac{e}{m^{2}}\right)^{2} \mathcal{G}$. Finally note that the action and the Lagrangian are trivially related in constant fields, differing only by an overall volume factor, i.e., $\Gamma_{\mathrm{HE}}=\mathcal{L}_{\mathrm{HE}} \int_{x}$.

In constant external fields, $\mathcal{L}_{\mathrm{HE}}^{1 \text {-loop }}$ and $\left.\mathcal{L}_{\mathrm{HE}}^{2 \text {-loop }}\right|_{1 \mathrm{PI}}$ are known explicitly in terms of parameter integral representations for both spinor [2, 65] and scalar [3, 67] QED; cf. [71] for a review. For instance, the on-shell renormalized one-loop effective Lagrangian for spinor QED is given by $[2,15]$,

$$
\mathcal{L}_{\mathrm{HE}}^{1-\text { loop }}=-\frac{1}{8 \pi^{2}} \int_{0}^{\infty} \frac{\mathrm{d} T}{T^{3}} \mathrm{e}^{-m^{2} T}\left\{\frac{(e \epsilon T)(e \eta T)}{\tan (e \epsilon T) \tanh (e \eta T)}-\frac{2}{3}(e T)^{2} \mathcal{F}-1\right\}
$$

where $\epsilon=\left(\sqrt{\mathcal{F}^{2}+\mathcal{G}^{2}}-\mathcal{F}\right)^{1 / 2}$ and $\eta=\left(\sqrt{\mathcal{F}^{2}+\mathcal{G}^{2}}+\mathcal{F}\right)^{1 / 2}$ are the secular invariants in constant electromagnetic fields. The analogous expression for $\left.\mathcal{L}_{\mathrm{HE}}^{2 \text {-loop }}\right|_{1 \mathrm{PI}}$ is given in eq. (B.11) in the appendix for spinor QED. For completeness, we also note that mass renormalization has to be taken into account from two loops on for diagrams involving fermion loops ith internal radiative corrections; see, e.g., [69].

As indicated above and determined explicitly below, the $1 \mathrm{PR}$ contribution $\left.\mathcal{L}_{\mathrm{HE}}^{2 \text {-loop }}\right|_{1 \mathrm{PR}}$ depicted in figure 4 is finite also in the constant field limit. Based on the structure of the 
LCFA, we detail below how the exact expression for $\left.\mathcal{L}_{\mathrm{HE}}^{\text {-loop }}\right|_{1 \mathrm{PR}}$ in constant fields can be inferred from the constant-field result for $\mathcal{L}_{\mathrm{HE}}^{\text {1-loop }}$, yielding

$$
\begin{aligned}
\left.\mathcal{L}_{\mathrm{HE}}^{2 \text {-loop }}\right|_{1 \mathrm{PR}} & =\frac{\partial \mathcal{L}_{\mathrm{HE}}^{1 \text {-loop }}}{\partial F^{\mu \nu}} \frac{\partial \mathcal{L}_{\mathrm{HE}}^{1 \text {-loop }}}{\partial F_{\mu \nu}} \\
& =\mathcal{F}\left[\left(\frac{\partial \mathcal{L}_{\mathrm{HE}}^{1-\text { loop }}}{\partial \mathcal{F}}\right)^{2}-\left(\frac{\partial \mathcal{L}_{\mathrm{HE}}^{1-\text { loop }}}{\partial \mathcal{G}}\right)^{2}\right]+2 \mathcal{G} \frac{\partial \mathcal{L}_{\mathrm{HE}}^{1 \text {-loop }}}{\partial \mathcal{F}} \frac{\partial \mathcal{L}_{\mathrm{HE}}^{1-\text { loop }}}{\partial \mathcal{G}} .
\end{aligned}
$$

In turn, $\left.\mathcal{L}_{\mathrm{HE}}^{2 \text {-loop }}\right|_{1 \mathrm{PR}}$ can be expressed in terms of a double parameter integral.

For illustration, let us concentrate on the weak and strong field asymptotics of $\mathcal{L}_{\mathrm{HE}}^{\text {1-loop }}$ and $\mathcal{L}_{\mathrm{HE}}^{\text {-loop }}=\left.\mathcal{L}_{\mathrm{HE}}^{2 \text {-loop }}\right|_{1 \mathrm{PI}}+\left.\mathcal{L}_{\mathrm{HE}}^{2 \text {-loop }}\right|_{1 \mathrm{PR}}$ for spinor QED. In the weak field limit, characterized by $\{\tilde{\mathcal{F}}, \tilde{\mathcal{G}}\} \ll 1$, the well-known literature results read $[1-3]$,

$$
\frac{\mathcal{L}_{\text {HE }}^{\text {1-loop }}}{m^{4}}=\frac{1}{4 \pi^{2}} \frac{1}{90}\left[\left(4 \tilde{\mathcal{F}}^{2}+7 \tilde{\mathcal{G}}^{2}\right)-\tilde{\mathcal{F}}\left(\frac{32}{7} \tilde{\mathcal{F}}^{2}+\frac{52}{7} \tilde{\mathcal{G}}^{2}\right)+\mathcal{O}\left(\epsilon^{8}\right)\right],
$$

and $[65]$

$$
\frac{\left.\mathcal{L}_{\text {HE }}^{2 \text { loop }}\right|_{1 \mathrm{PI}}}{m^{4}}=\frac{\alpha}{\pi} \frac{1}{4 \pi^{2}} \frac{1}{90}\left[\left(\frac{160}{9} \tilde{\mathcal{F}}^{2}+\frac{1315}{36} \tilde{\mathcal{G}}^{2}\right)-\tilde{\mathcal{F}}\left(\frac{1219}{45} \tilde{\mathcal{F}}^{2}+\frac{2164}{45} \tilde{\mathcal{G}}^{2}\right)+\mathcal{O}\left(\epsilon^{8}\right)\right],
$$

where we count $\mathcal{O}\left(\frac{e \bar{F}^{\mu \nu}}{m^{2}}\right) \sim \mathcal{O}(\epsilon)$. The terms given explicitly in eqs. (3.3) and (3.4) amount to the 1PI diagrams depicted in figures 1 and 4 with the fermion loop featuring four and six couplings to the external field, respectively. For the two-loop 1PR contribution, we obtain from eq. (3.2) the new result

$$
\frac{\left.\mathcal{L}_{\mathrm{HE}}^{2 \text {-loop }}\right|_{1 \mathrm{PR}}}{m^{4}}=\frac{\alpha}{\pi} \frac{1}{4 \pi^{2}} \frac{1}{90}\left[\tilde{\mathcal{F}}\left(\frac{32}{45} \tilde{\mathcal{F}}^{2}+\frac{14}{45} \tilde{\mathcal{G}}^{2}\right)+\mathcal{O}\left(\epsilon^{8}\right)\right]
$$

The contribution given explicitly here stems from the 1PR diagram in figure 4 with each fermion loop exhibiting three couplings to the external field. For $|\tilde{\mathcal{F}}| \gg 1$ and $|\tilde{\mathcal{G}}| \ll 1$ corresponding to the cases of strong electric or magnetic fields, we obtain (for the derivation, see appendix B)

$$
\frac{\mathcal{L}_{\text {HE }}^{1 \text { loop }}}{m^{4}}=\frac{1}{4 \pi^{2}} \frac{1}{3}\left\{\tilde{\mathcal{F}}\left[\ln \sqrt{\tilde{\mathcal{F}}}+\mathcal{O}\left(\left(\frac{1}{\tilde{\mathcal{F}}}\right)^{0}\right)\right]+\frac{1}{2 \sqrt{2}} \frac{\tilde{\mathcal{G}}^{2}}{\tilde{\mathcal{F}}}\left[\sqrt{\tilde{\mathcal{F}}}+\mathcal{O}\left(\left(\frac{1}{\tilde{\mathcal{F}}}\right)^{0}\right)\right]+\mathcal{O}\left(\tilde{\mathcal{G}}^{4}\right)\right\}
$$

and

$\frac{\left.\mathcal{L}_{\text {HE }}^{2 \text {-loop }}\right|_{1 \mathrm{PI}}}{m^{4}}=\frac{\alpha}{\pi} \frac{1}{4 \pi^{2}} \frac{1}{4}\left\{\tilde{\mathcal{F}}\left[\ln \sqrt{\tilde{\mathcal{F}}}+\mathcal{O}\left(\left(\frac{1}{\tilde{\mathcal{F}}}\right)^{0}\right)\right]-\frac{1}{3 \sqrt{2}} \frac{\tilde{\mathcal{G}}^{2}}{\tilde{\mathcal{F}}}\left[\sqrt{\tilde{\mathcal{F}}}+\mathcal{O}\left(\left(\frac{1}{\tilde{\mathcal{F}}}\right)^{0}\right)\right]+\mathcal{O}\left(\tilde{\mathcal{G}}^{4}\right)\right\}$,

where

$$
\sqrt{\tilde{\mathcal{F}}}=\sqrt{|\tilde{\mathcal{F}}|}\{\Theta(\tilde{\mathcal{F}})-i \Theta(-\tilde{\mathcal{F}})\} .
$$

In addition to the well-known leading-log terms [4, 65, 67, 72], eqs. (3.6) and (3.7) also account for the strongly suppressed contribution $\sim \tilde{\mathcal{G}}^{2}$ which is of relevance for the photon 
polarization tensor (cf. section 4.4 below). Note that this contribution is suppressed as $\sim \tilde{\mathcal{G}}^{2} / \sqrt{\tilde{\mathcal{F}}}$, such that the criterion $|\tilde{\mathcal{G}}| \ll 1$ imposed for the expansion seems actually rather conservative, and it might be sufficient to demand $\tilde{\mathcal{G}}^{2} / \sqrt{\tilde{\mathcal{F}}} \ll 1$ instead. However, we have not analyzed the scaling of any terms at $\mathcal{O}\left(\tilde{\mathcal{G}}^{4}\right)$. Apart from an overall parametric suppression of $\left.\mathcal{L}_{\mathrm{HE}}^{2 \text {-loop }}\right|_{1 \mathrm{PI}}$ by a factor of $\frac{\alpha}{\pi}$, the weak and strong field limits of $\mathcal{L}_{\mathrm{HE}}^{1 \text {-loop }}$ and $\left.\mathcal{L}_{\mathrm{HE}}^{2 \text {-loop }}\right|_{1 \mathrm{PI}}$ are of the same structure and only differ in the specific numerical coefficients. By contrast, the $1 \mathrm{PR}$ contribution to $\mathcal{L}_{\mathrm{HE}}^{2 \text {-loop }}$ scales as

$$
\frac{\left.\mathcal{L}_{\text {HE }}^{2 \text {-loop }}\right|_{1 \mathrm{PR}}}{m^{4}}=\frac{\alpha}{\pi} \frac{1}{4 \pi^{2}} \frac{1}{3}\left\{\tilde{\mathcal{F}}\left[\frac{1}{3} \ln ^{2} \sqrt{\tilde{\mathcal{F}}}-\left(1-8 \zeta^{\prime}(-1)\right) \ln \sqrt{\tilde{\mathcal{F}}}+\mathcal{O}\left(\left(\frac{1}{\tilde{\mathcal{F}}}\right)^{0}\right)\right]+\mathcal{O}\left(\tilde{\mathcal{G}}^{2}\right)\right\},
$$

from which we infer $\left(\left.\mathcal{L}_{\mathrm{HE}}^{2 \text {-loop }}\right|_{1 \mathrm{PR}}\right) /\left(\left.\mathcal{L}_{\mathrm{HE}}^{2 \text {-loop }}\right|_{1 \mathrm{PI}}\right) \sim \frac{4}{9} \ln \sqrt{\tilde{\mathcal{F}}}$, implying that $\left.\mathcal{L}_{\mathrm{HE}}^{2 \text {-loop }}\right|_{1 \mathrm{PR}}$ dominates over $\left.\mathcal{L}_{\mathrm{HE}}^{\text {-loop }}\right|_{1 \mathrm{PI}}$ in this limit. This dominance due to the occurrence of a squared logarithm is a direct consequence of the $1 \mathrm{PR}$ structure. For completeness, also note that $\mathcal{L}_{\mathrm{HE}}^{2 \text {-loop }} / \mathcal{L}_{\mathrm{HE}}^{1 \text {-loop }} \sim \frac{\alpha}{\pi} \frac{1}{3} \ln \sqrt{\tilde{\mathcal{F}}}$. The criterion of apparent convergence of the loop expansion hence suggests the breakdown of the perturbative loop expansion for the Heisenberg-Euler action at exponentially large fields.

Apart from these constant-field results, only a few exact results for $\Gamma_{\mathrm{HE}}^{1-l o o}$ in specific (one-dimensional) field inhomogeneities are known explicitly; cf., e.g., [73-76], and [71] for a review. Also note that the effective action vanishes identically for the case of a single monochromatic plane wave field [15]. On the three-loop level, first analytical results for the 1PI part of $\Gamma_{\mathrm{HE}}^{3 \text {-loop }}$ have been obtained in $1+1$ dimensions $[77,78]$. No further analytical results for $\Gamma_{\mathrm{HE}}^{l-\text { loop }}$ with $l>2$ as well as for more-dimensional field inhomogeneities are available so far.

\section{Effective theory of low-frequency photons in slowly varying electro- magnetic fields}

\subsection{Locally constant field approximation}

In the spirit of the LCFA, the Heisenberg-Euler effective action for constant fields can also be adopted for slowly varying inhomogeneous fields. The LCFA amounts to substituting $\bar{F}^{\mu \nu} \rightarrow \bar{F}^{\mu \nu}(x)$ in the constant-field result for the Lagrangian, such that $\mathcal{L}_{\mathrm{HE}}\left(\mathcal{F}, \mathcal{G}^{2}\right) \rightarrow$ $\mathcal{L}_{\mathrm{HE}}\left(\mathcal{F}(x), \mathcal{G}^{2}(x)\right)$. In turn, the corresponding action becomes a functional of a varying field $\bar{F}^{\mu \nu}(x)$, i.e., $\Gamma_{\mathrm{HE}}\left[\mathcal{F}(x), \mathcal{G}^{2}(x)\right]=\int_{x} \mathcal{L}_{\mathrm{HE}}\left(\mathcal{F}(x), \mathcal{G}^{2}(x)\right)$.

The deviations of this LCFA result from the corresponding - typically unknown exact result for $\Gamma_{\mathrm{HE}}$ in the particular inhomogeneous background field profile under consideration are of order $\mathcal{O}\left(\left(\frac{v}{m}\right)^{2}\right)$, where $v$ delimits the moduli of the frequency and momentum components of the considered inhomogeneous field from above [68, 79]. The reasoning to arrive at this conclusion is as follows: as $\Gamma_{\mathrm{HE}}$ is both a Lorentz scalar and a gauge invariant quantity, and the associated Lagrangian should be "almost local" for slowly varying fields, its dependence on the external field $\bar{A}^{\mu}(x)$ should be expressible in terms of $\bar{F}^{\mu \nu}(x)$, ${ }^{*} \bar{F}^{\mu \nu}(x)$ and derivatives thereof. Any scalar quantity made up of combinations of $\bar{F},{ }^{*} \bar{F}$ 


$$
\Gamma_{\text {int }}=O+m+m+q^{r}
$$

Figure 5. Graphical representation of $\Gamma_{\text {int }}=\sum_{n=0}^{\infty} \Gamma_{\text {int }}^{(n)}$. The gray bubbles symbolize the effective couplings of $n$ low-frequency photon fields $a(x)$; generically they are made up of 1PI diagrams of arbitrary loop order. In momentum space they are given by $s_{(n)}^{\sigma_{1} \ldots \sigma_{n}}\left(k_{1}, \ldots, k_{n}\right)$ defined in eq. (4.5).

and $\partial$ is necessarily even in $\partial$. Canonical power-counting implies that the occurrence of any derivative $\partial$ has to be balanced by a dimensionful scale. In QED and for generic laboratory fields, this scale is provided by the electron mass $m$, leading to the above criterion. This implies that the LCFA constitutes a good approximation for inhomogeneous fields fulfilling $v \ll m$. In position space this criterion translates to the requirement that the inhomogeneous fields under consideration should only vary on scales much larger than the Compton wavelength $\lambda$ and time $\lambda / c$ of the electron; cf. section 1. Explicit results for higher orders in the derivative expansion show, that the dimensional balancing of derivatives can also be taken over by the field strength itself for strong fields $|e \bar{F}(x)| \gg m^{2}$, thereby increasing the validity range of the LCFA in that regime [80].

\subsection{Effective action for low-frequency photons}

In a next step, we employ the LCFA result for the 1PI part of $\Gamma_{\mathrm{HE}}$ as an effective action $\Gamma_{\text {eff }}$, describing the propagation and interactions of dynamical low-frequency photon fields in the quantum vacuum subject to the slowly varying external field. More precisely, we define this effective action as

$$
\Gamma_{\text {eff }}[a(x), \bar{F}(x)]:=-\frac{1}{4} \int_{x} f_{\mu \nu} f^{\mu \nu}+\underbrace{\left.\left(\left.\Gamma_{\mathrm{HE}}\left[\mathcal{F}(x), \mathcal{G}^{2}(x)\right]\right|_{1 \mathrm{PI}}+\int_{x} \mathcal{F}(x)\right)\right|_{\bar{F} \rightarrow \bar{F}+f}}_{=: \Gamma_{\text {int }}[a(x), \bar{F}(x)]}
$$

where $\left.\Gamma_{\mathrm{HE}}\right|_{1 \mathrm{PI}}$ denotes the 1PI part of the Heisenberg-Euler effective action, and the field strength tensor $\bar{F}$ is understood to be shifted as follows [19, 68, 79],

$$
\bar{F}(x) \rightarrow \bar{F}(x)+f(x) .
$$

After this shift, $\bar{F}(x)$ describes the slowly varying external field with $v \ll m$, and $f^{\mu \nu}(x)=$ $\partial^{\mu} a^{\nu}(x)-\partial^{\nu} a^{\mu}(x)$ is to be interpreted as the field strength tensor of a dynamical photon field $a^{\mu}(x)=\int_{p} e^{i p x} a^{\mu}(p)$, with $a^{\mu}(p)$ receiving all its relevant contributions from the momentum regime where $\left\{\left|p^{0}\right|,|\vec{p}|\right\} \lesssim v \ll m$. In many cases of physical interest, $\bar{F}(x)$ plays the role of the applied field and $a(x)$ that of a signal field as introduced in eq. (2.9).

It is then convenient to organize $\Gamma_{\text {int }}$ in terms of interactions involving $n \in \mathbb{N}_{0}$ photon fields, i.e., $\Gamma_{\text {int }}=\sum_{n=0}^{\infty} \Gamma_{\text {int }}^{(n)}$, with $\Gamma_{\text {int }}^{(n)} \equiv \Gamma_{\text {int }}^{(n)}\left[a(x), \mathcal{F}(x), \mathcal{G}^{2}(x)\right] \sim a^{n}$. For a graphical representation of this expansion, cf. figure 5. More specifically, we have

$$
\Gamma_{\mathrm{int}}^{(n)}=\frac{1}{n !} \int_{x} \prod_{j=1}^{n}\left(f^{\mu_{j} \nu_{j}}(x) \frac{\partial}{\partial \bar{F}^{\mu_{j} \nu_{j}}(x)}\right)\left(\left.\mathcal{L}_{\mathrm{HE}}\left(\mathcal{F}(x), \mathcal{G}^{2}(x)\right)\right|_{1 \mathrm{PI}}+\mathcal{F}(x)\right),
$$


which implies that $\left.\mathcal{L}_{\mathrm{HE}}\left(\mathcal{F}(x), \mathcal{G}^{2}(x)\right)\right|_{1 \mathrm{PI}}$ generates effective photon interactions to any order in $n[79,81]$. As the LCFA results in a local Lagrangian, by construction all these effective interactions are local with respect to $f^{\mu \nu}(x)$, and correspondingly in $a^{\mu}(x)$. Let us also emphasize that by construction we have to assume that the combination of any given number $n$ of low-frequency photons again gives rise to a low-frequency photon. For the following discussion it is more convenient to turn to momentum space where the derivatives acting on the photon fields contained in $f^{\mu \nu}(x)$ translate into multiplicative factors of the associated momenta, i.e., $f^{\mu \nu}(x)=i \int_{p} e^{i p x} a_{\sigma}(p)\left[p^{\mu} g^{\nu \sigma}-p^{\nu} g^{\mu \sigma}\right]$. This results in

$$
\Gamma_{\text {int }}^{(n)}=\frac{1}{n !} \prod_{j=1}^{n}\left(\int_{p_{j}} a_{\sigma_{j}}\left(p_{j}\right)\right) s_{(n)}^{\sigma_{1} \ldots \sigma_{n}}\left(p_{1}, \ldots, p_{n}\right),
$$

with the effective $n$ photon couplings $s_{(n)}^{\sigma_{1} \ldots \sigma_{n}}\left(p_{1}, \ldots, p_{n}\right)$ (1PI proper vertices) given by

$$
\begin{aligned}
& s_{(n)}^{\sigma_{1} \ldots \sigma_{n}}\left(p_{1}, \ldots, p_{n}\right) \\
& \quad=(2 i)^{n} \int_{x} e^{i x \sum_{j=1}^{n} p_{j}} \prod_{j=1}^{n}\left(p_{j}^{\mu_{j}} g^{\nu_{j} \sigma_{j}} \frac{\partial}{\partial \bar{F}^{\mu_{j} \nu_{j}}(x)}\right)\left(\left.\mathcal{L}_{\mathrm{HE}}\left(\mathcal{F}(x), \mathcal{G}^{2}(x)\right)\right|_{1 \mathrm{PI}}+\mathcal{F}(x)\right) .
\end{aligned}
$$

The latter obviously fulfill the Ward identity $\left(p_{j}\right)_{\sigma_{j}} s_{(n)}^{\sigma_{1} \ldots \sigma_{j} \ldots \sigma_{n}}\left(p_{1}, \ldots, p_{j}, \ldots, p_{n}\right)=0$ for any fixed value of $1 \leq j \leq n$.

For $\bar{F}=$ const. the external field cannot absorb or supply momentum, and the $x$ integration in eq. (4.5) can be performed right away, resulting in an overall delta function, $\int_{x} e^{i x \sum_{j=1}^{n} p_{j}}=(2 \pi)^{4} \delta\left(\sum_{j=1}^{n} p_{j}^{\mu}\right)$, ensuring four-momentum conservation in the effective coupling of $n$ photons. Hence, in this limit the effective $n$ photon interactions are of the same momentum structure as at zero external field. However, for $\bar{F}=$ const. $\neq 0$, also effective couplings involving an odd number of photons are induced. This is in contrast to the zero-field case, where fermion loops with an odd number of photon couplings of course vanish identically because of Furry's theorem.

The contribution $s_{(1)}^{\sigma_{1}}\left(p_{1}\right)$ in eq. (4.5) constitutes a photon current [63, 82] and $s_{(2)}^{\sigma_{1} \sigma_{2}}\left(p_{1}, p_{2}\right)$ a photon polarization tensor [68]. In more conventional notations, the quantum corrections to the effective action up to quadratic order in $a^{\mu}$ are given by

$$
\Gamma_{\mathrm{int}}=\Gamma_{\mathrm{int}}^{(0)}+\int_{p} a_{\sigma}(p) j^{\sigma}(p)+\frac{1}{2} \int_{p} \int_{p^{\prime}} a_{\rho}(p) s_{(2)}^{\rho \sigma}\left(p, p^{\prime}\right) a_{\sigma}\left(p^{\prime}\right)+\mathcal{O}\left(a^{3}\right),
$$

with $j^{\sigma}(p):=s_{(1)}^{\sigma}(p)$. The neglected higher-order terms of $\mathcal{O}\left(a^{3}\right)$ correspond to effective interactions involving three or more photons, giving rise to, e.g., direct light-bylight scattering $[1,83,84]$, photon splitting [19, 52, 54, 55] and higher-harmonic generation $[61,62,85,86]$.

Obviously no real (on-shell) photons can be generated from constant external fields, as

$$
\left.j^{\sigma}(p)\right|_{\bar{F}=\text { const. }} \sim \int_{x} e^{i x p} p^{\sigma}=(2 \pi)^{4} \delta(p) p^{\sigma} .
$$

The physical reason for this is that a constant external field cannot supply momentum to the virtual charged particle-antiparticle fluctuations. Still, the fields $a^{\mu}$ can be propagating fields, the free causal propagation of which is described by the usual Feynman 
propagator (2.25). Within the LCFA, we have the additional constraint that the considered momentum transfer is manifestly restricted to the soft momentum regime, i.e., $\left\{\left|p^{0}\right|,|\vec{p}|\right\} \lesssim v \ll m$ (cf. above).

Here, we argue that this constraint will be fulfilled automatically in the evaluation of all the Feynman diagrams that can arise as quantum corrections within the effective theory $\Gamma_{\text {eff }}$ of low-frequency photon fields in slowly varying electromagnetic fields. For this, we first stress that $\Gamma_{\text {eff }}$ already incorporates all 1PI proper vertices by definition, such that further quantum corrections to be evaluated within the effective theory of low-frequency photon fields must be 1PR. By construction, the virtual photons in these 1PR diagrams mediate between slowly varying fields only, since the external lines of the 1PI building blocks are either low-frequency photons or slowly varying electromagnetic fields. Hence, the above kinematic constraint is indeed fulfilled automatically.

In a next step, we utilize $\Gamma_{\text {eff }}$ to derive some physically relevant explicit results: as the prime example, we compute the 1PR contribution to the Heisenberg-Euler effective action $\left.\Gamma_{\mathrm{HE}}^{2 \text {-loops }}\right|_{1 \mathrm{PR}}$ in slowly varying external fields, introduced and discussed already in sections 2 and 3 above.

\subsection{PR contribution to the Heisenberg-Euler effective action}

Let us now focus on the effective self-interactions of the external electromagnetic field arising in this theory. At one-loop order these are encoded in $\left.\Gamma_{\text {int }}^{(0)}\right|_{1-\text { loop }}=\Gamma_{\mathrm{HE}}^{1 \text {-loop }}$ (cf. figures 1 and 5). At two loops, in addition to $\left.\Gamma_{\text {int }}^{(0)}\right|_{2 \text {-loop }}=\left.\Gamma_{\mathrm{HE}}^{2 \text {-loop }}\right|_{1 \mathrm{PI}}$, also the $1 \mathrm{PR}$ diagram depicted in figure 4 (right) contributes. It corresponds to the following expression:

$$
\left.\Gamma_{\mathrm{HE}}^{2 \text {-loop }}\right|_{1 \mathrm{PR}}=\int_{p} j_{1 \text {-loop }}^{\mu}(p) D_{\mu \nu}(p) j_{1 \text {-loop }}^{\nu}(-p),
$$

where $j_{l \text {-loop }}^{\mu}:=\left.s_{(1)}^{\mu}\right|_{l \text {-loop }}$. We emphasize that the integration in eq. (4.8), which is formally over all virtual momentum transfers, exclusively receives contributions from the soft momentum regime. This is because the photon currents $j^{\mu}(p)$ only induce low-energy modes by construction via the LCFA. The constant-field limit in eq. (4.7) provides an obvious example for the underlying mechanism. Inserting the explicit expressions for the currents and the photon propagator (2.25) in the Feynman gauge, we obtain

$$
\left.\Gamma_{\mathrm{HE}}^{2 \text {-loop }}\right|_{1 \mathrm{PR}}=\int_{x} \int_{x^{\prime}} G^{\mu \nu}\left(x-x^{\prime}\right) \frac{\partial \mathcal{L}_{\mathrm{HE}}^{1-\text { loop }}}{\partial \bar{F}^{\mu}{ }_{\alpha}}(x) \frac{\partial \mathcal{L}_{\mathrm{HE}}^{1-\text { loop }}}{\partial \bar{F}^{\nu \alpha}}\left(x^{\prime}\right) .
$$

Here we have defined

$$
G^{\mu \nu}(\tilde{x}):=4 \int_{p} \frac{p^{\mu} p^{\nu}}{p^{2}-i \epsilon} e^{i \tilde{x} p}=\frac{2}{\pi^{2}} \frac{i}{\left(\tilde{x}^{2}+i \epsilon\right)^{2}}\left(g^{\mu \nu}-4 \frac{\tilde{x}^{\mu} \tilde{x}^{\nu}}{\tilde{x}^{2}+i \epsilon}\right),
$$

which fulfills $\frac{1}{4} g_{\mu \nu} G^{\mu \nu}(\tilde{x})=\delta(\tilde{x})$ and $\int_{\tilde{x}} G^{\mu \nu}(\tilde{x})=g^{\mu \nu}$. 
Expressing the derivatives for $\bar{F}$ in terms of derivatives for $\mathcal{F}$ and $\mathcal{G}$ (cf. appendix A), eq. (4.9) can be represented as

$$
\begin{aligned}
\left.\Gamma_{\mathrm{HE}}^{2 \text {-loop }}\right|_{1 \mathrm{PR}}= & \left\{\frac{1}{2} \int_{x} \int_{x^{\prime}} G_{\alpha \beta}\left(x-x^{\prime}\right) \bar{F}_{\rho}^{\alpha}(x)\right. \\
& \times\left[\frac{1}{2} \bar{F}^{\beta \rho}\left(x^{\prime}\right)\left(\frac{\partial \mathcal{L}}{\partial \mathcal{F}}(x) \frac{\partial \mathcal{L}}{\partial \mathcal{F}}\left(x^{\prime}\right)+\frac{\partial \mathcal{L}}{\partial \mathcal{G}}(x) \frac{\partial \mathcal{L}}{\partial \mathcal{G}}\left(x^{\prime}\right)\right)+{ }^{*} \bar{F}^{\beta \rho}\left(x^{\prime}\right) \frac{\partial \mathcal{L}}{\partial \mathcal{F}}(x) \frac{\partial \mathcal{L}}{\partial \mathcal{G}}\left(x^{\prime}\right)\right] \\
& \left.-2 \int_{x} \mathcal{F}(x)\left(\frac{\partial \mathcal{L}}{\partial \mathcal{G}}(x)\right)^{2}\right\}\left.\right|_{\mathcal{L}=\mathcal{L}_{\mathrm{HE}}^{\text {1-loop }}},
\end{aligned}
$$

where we employed the identity ${ }^{*} \bar{F}_{\rho}^{\alpha}(x){ }^{*} \bar{F}^{\beta \rho}\left(x^{\prime}\right)=\bar{F}^{\beta \rho}(x) \bar{F}_{\rho}^{\alpha}\left(x^{\prime}\right)-\frac{1}{2} g^{\alpha \beta} \bar{F}_{\sigma \rho}(x) \bar{F}^{\sigma \rho}\left(x^{\prime}\right)$. The products of derivatives of $\mathcal{L}_{\mathrm{HE}}^{1 \text {-loop }}$ in eq. (4.11) for $\mathcal{F}$ and $\mathcal{G}$ can be expressed in terms of double integral representations which follow directly from the parameter integral representation of $\mathcal{L}_{\text {HE }}^{1 \text {-loop }}$.

Even though derived from a LCFA, eq. (4.11) gives rise to nonlocal interactions among electromagnetic fields. However, for slowly varying electromagnetic fields as considered here, these nonlocalities are expected to be very weak. Particularly for constant external fields, the field strength tensor $\bar{F}$ and thus the effective Lagrangian become independent of $x$ and $x^{\prime}$, such that the integrations over position space in eqs. (4.9) and (4.11) can be performed right away, resulting in eq. (3.2) above.

Let us finally resolve the seeming discrepancy that the constant-field limit of eqs. (4.9) and (4.11) yields the finite result (3.2) even though the formal expression of the photon current vanishes in constant fields; cf. eq. (4.7). The photon current (4.7) vanishes because of the factor linear in $p^{\sigma}$ multiplying $\delta(p)$, which ensures the Ward identity $p_{\sigma} j^{\sigma}(p)=0$ to hold. This implies that no real external photons $a_{\sigma}(p)$, exhibiting a regular behavior for $p^{\sigma} \rightarrow 0$, can be induced from $j^{\sigma}(p)$. However, this behavior is clearly not fulfilled by virtual photons. As the photon propagator $(2.25)$ scales as $\sim \frac{1}{p^{2}}$, the linear momentum dependences of the two individual currents effectively drop out upon combination with two photon currents, leaving us with a finite contribution.

Correspondingly, the two-loop physical effective interaction among generic external electromagnetic fields is determined by the combination $\Gamma_{\mathrm{HE}}^{2 \text {-loop }}=\left.\Gamma_{\mathrm{HE}}^{2 \text {-loo }}\right|_{1 \mathrm{PI}}+\left.\Gamma_{\mathrm{HE}}^{2 \text {-loop }}\right|_{1 \mathrm{PR}}$. Of course, similar 1PR diagrams are expected to contribute to the self-interactions of the external electromagnetic field at higher loop orders - even in constant external fields. Besides, they obviously also need to be accounted for in determining the effective interactions between any given number of photons in the quantum vacuum subject to external electromagnetic fields.

\subsection{Low-frequency photon propagation}

In this section we study quantum corrections to photon propagation - i.e., photon-photon correlators - in external electromagnetic fields up to order $\left(\frac{\alpha}{\pi}\right)^{2}$. It is instructive to have a look on the various Feynman diagrams potentially contributing to photon propagation up to this order; see figure 5. We organize them into (a) 1PI diagrams, (b) 1PR diagrams, and disconnected contributions which amount to (c) current-current correlators. Note that 
(a) one-particle irreducible:<smiles>Cc1ccccc1</smiles>
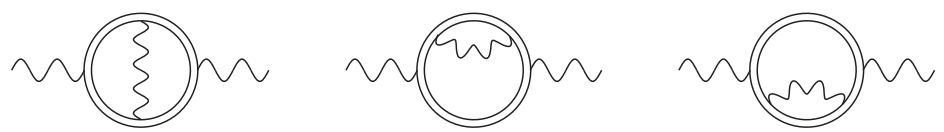

(b) one-particle reducible:

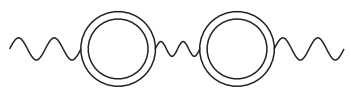<smiles>COc1cccc(-c2ccccc2)c1</smiles><smiles>Cc1cccc(-c2ccccc2)c1</smiles>

(c) current-current correlators:

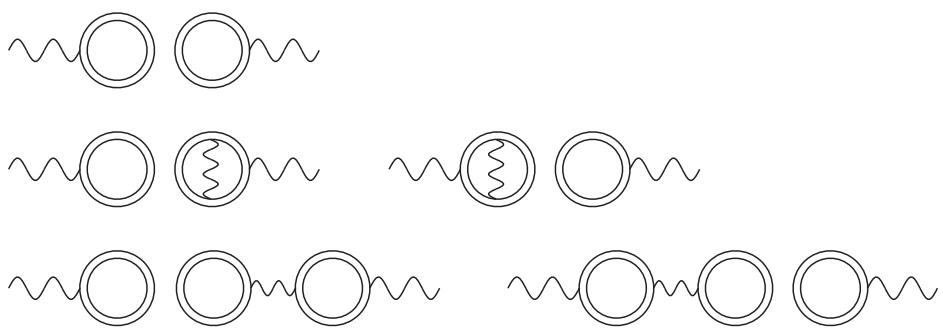

Figure 6. Different types of Feynman diagrams contributing to photon propagation in generic external electromagnetic fields at order $\frac{\alpha}{\pi}$ and $\left(\frac{\alpha}{\pi}\right)^{2}$; for the definition of the double line, cf. figure 1 . Note that the presence of the current-current diagrams (c) invalidates the equivalence between counting numbers of loops and powers of $\frac{\alpha}{\pi}$.

there are just two diagrams at order $\frac{\alpha}{\pi}$, namely the first one in figure $6(\mathrm{a})$ and in figure $6(\mathrm{c})$, respectively. All the other diagrams shown are proportional to $\left(\frac{\alpha}{\pi}\right)^{2}$.

The diagrams depicted in figure 6(a) constitute the 1PI part of the photon polarization tensor at one (first line) and two loops (second line). They are contained in $\Gamma_{\text {int }}^{(2)}$, and are given by

$$
\left.s_{(2)}^{\rho \sigma}\left(p, p^{\prime}\right)\right|_{l \text {-loop }}=-\left.4 p^{\mu} p^{\nu} \int_{x} e^{i\left(p+p^{\prime}\right) x} \frac{\partial^{2} \mathcal{L}_{\mathrm{HE}}^{l \text {-loop }}}{\partial \bar{F}^{\mu}{ }_{\rho} \partial \bar{F}^{\nu}{ }_{\sigma}}(x)\right|_{1 \mathrm{PI}} ;
$$

cf. eqs. (4.4)-(4.6). Note that $\left.s_{(2)}^{\rho \sigma}\right|_{1-\text { loop }}$ corresponds to the slowly varying field limit of the one-loop polarization tensor $\left.\Pi^{\rho \sigma} \equiv s_{(2)}^{\rho \sigma}\right|_{1 \text {-loop }}$ defined in section 2.3 above. The definition (4.12) automatically accounts for all the topologically inequivalent 1PI diagrams depicted in the second line of figure 6(a). Obviously, we have $\left.s_{(2)}^{\rho \sigma}\left(p, p^{\prime}\right)\right|_{l \text {-loop }} \sim\left(\frac{\alpha}{\pi}\right)^{l}$. Expressing the derivatives for $\bar{F}$ in terms of derivatives for $\mathcal{F}$ and $\mathcal{G}$, eq. (4.12) can be 
represented as [68]

$$
\begin{aligned}
\left.s_{(2)}^{\rho \sigma}\left(p, p^{\prime}\right)\right|_{l \text {-loop }}= & -\int_{x} e^{i\left(p+p^{\prime}\right) x}\left[\left(\left(p p^{\prime}\right) g^{\rho \sigma}-p^{\prime \rho} p^{\sigma}\right) \frac{\partial \mathcal{L}}{\partial \mathcal{F}}(x)+p_{\mu}^{\prime} p_{\alpha} \epsilon^{\rho \sigma \mu \alpha} \frac{\partial \mathcal{L}}{\partial \mathcal{G}}(x)\right. \\
& +(p \bar{F}(x))^{\rho}\left(p^{\prime} \bar{F}(x)\right)^{\sigma} \frac{\partial^{2} \mathcal{L}}{\partial \mathcal{F}^{2}}(x)+\left(p^{*} \bar{F}(x)\right)^{\rho}\left(p^{\prime *} \bar{F}(x)\right)^{\sigma} \frac{\partial^{2} \mathcal{L}}{\partial \mathcal{G}^{2}}(x) \\
& \left.+\left[\left(p^{*} \bar{F}(x)\right)^{\rho}\left(p^{\prime} \bar{F}(x)\right)^{\sigma}+(p \bar{F}(x))^{\rho}\left(p^{\prime *} \bar{F}(x)\right)^{\sigma}\right] \frac{\partial^{2} \mathcal{L}}{\partial \mathcal{F} \partial \mathcal{G}}(x)\right]\left.\right|_{\mathcal{L}=\left.\mathcal{L}_{\text {HE }}^{l \text {-loop }}\right|_{1 \mathrm{PI}},},
\end{aligned}
$$

where we have employed the shorthand notations $(p \bar{F})^{\mu}=p_{\nu} \bar{F}^{\nu \mu},\left(p^{*} \bar{F}\right)^{\mu}=p_{\nu}{ }^{*} \bar{F}^{\nu \mu}$, $\left(p p^{\prime}\right)=p_{\mu} p^{\prime \mu}$, etc. In the constant-field limit, $\bar{F}$ as well as $\mathcal{L}_{\mathrm{HE}}$ do not depend on the space-time coordinate and eq. (4.14) simplifies significantly: in this limit, the $x$ integration can be performed right away, giving rise to an overall delta function ensuring momentum conservation in constant fields, and thus

$$
\left.s_{(2)}^{\rho \sigma}\left(p, p^{\prime}\right)\right|_{l \text {-loop }}=(2 \pi)^{4} \delta\left(p+p^{\prime}\right) \sum_{j=0}^{3} P_{j}^{\rho \sigma}(p) c_{j}^{l-\text { loop }}(\mathcal{F}, \mathcal{G}),
$$

where we have introduced the tensor structures

$$
\begin{aligned}
& P_{0}^{\rho \sigma}(p)=\left(p^{2} g^{\rho \sigma}-p^{\rho} p^{\sigma}\right) \\
& P_{1}^{\rho \sigma}(p)=(p \bar{F})^{\rho}(p \bar{F})^{\sigma} \\
& P_{2}^{\rho \sigma}(p)=\left(p^{*} \bar{F}\right)^{\rho}\left(p^{*} \bar{F}\right)^{\sigma} \\
& P_{3}^{\rho \sigma}(p)=\left[\left(p^{*} \bar{F}\right)^{\rho}(p \bar{F})^{\sigma}+(p \bar{F})^{\rho}\left(p^{*} \bar{F}\right)^{\sigma}\right] .
\end{aligned}
$$

The associated coefficients $c_{j}^{l \text {-loop }}(\mathcal{F}, \mathcal{G})$ are given by

$$
\begin{array}{ll}
c_{0}^{l \text {-loop }}=\left.\frac{\partial \mathcal{L}_{\mathrm{HE}}^{l \text {-loop }}}{\partial \mathcal{F}}\right|_{1 \mathrm{PI}}, & c_{1}^{l \text {-loop }}=\left.\frac{\partial^{2} \mathcal{L}_{\mathrm{HE}}^{l \text {-loop }}}{\partial \mathcal{F}^{2}}\right|_{1 \mathrm{PI}}, \\
c_{2}^{l \text {-loop }}=\left.\frac{\partial^{2} \mathcal{L}_{\mathrm{HE}}^{l \text {-loop }}}{\partial \mathcal{G}^{2}}\right|_{1 \mathrm{PI}}, & c_{3}^{l \text {-loop }}=\left.\frac{\partial^{2} \mathcal{L}_{\mathrm{HE}}^{l \text {-loop }}}{\partial \mathcal{F} \partial \mathcal{G}}\right|_{1 \mathrm{PI}} .
\end{array}
$$

Note that the tensor structure $p_{\mu}^{\prime} p_{\alpha} \epsilon^{\rho \sigma \mu \alpha}$ vanishes in constant fields, where $p_{\mu}^{\prime}=-p_{\mu}$.

Let us now have a closer look on the other Feynman diagrams depicted in figure 6 . The first diagram in figure $6(\mathrm{~b})$ is just an iteration of $\Pi^{\rho \sigma}$, and the corresponding expression reads

$$
\begin{aligned}
\int_{k} \Pi^{\rho \mu}(p, k) & D_{\mu \nu}(k) \Pi^{\nu \sigma}\left(-k, p^{\prime}\right) \\
& =4 p^{\alpha} p^{\prime \beta} \int_{x} \int_{x^{\prime}} e^{i\left(x p+x^{\prime} p^{\prime}\right)} G^{\mu \nu}\left(x-x^{\prime}\right) \frac{\partial^{2} \mathcal{L}_{\mathrm{HE}}^{1-\text { loop }}}{\partial \bar{F}_{\rho}^{\alpha}{ }_{\rho} \bar{F}_{\sigma}^{\mu}}(x) \frac{\partial^{2} \mathcal{L}_{\mathrm{HE}}^{1-\text { loop }}}{\partial \bar{F}^{\sigma \nu} \partial \bar{F}_{\sigma}^{\beta}}\left(x^{\prime}\right) .
\end{aligned}
$$

A similar diagram exists in the absence of external fields. Contrarily, all the other diagrams in figure 6(b) and those in figure 6(c) do not contribute at zero field, because of Furry's theorem. The last two diagrams in figure 6(b) arise from saturating one leg of an effective 
three-photon coupling with $j_{1 \text {-loop }}$. Both diagrams yield the same result. Their combined contribution is given by

$$
\begin{aligned}
\left.2 \int_{k} s_{(3)}^{\rho \sigma \mu}\left(p, p^{\prime},-k\right)\right|_{1-\text { loop }} D_{\mu \nu}(k) j_{\text {1-loop }}^{\nu}(k) & \\
& =8 p^{\alpha} p^{\prime \beta} \int_{x} \int_{x^{\prime}} e^{i x\left(p+p^{\prime}\right)} G^{\mu \nu}\left(x-x^{\prime}\right) \frac{\partial^{3} \mathcal{L}_{\mathrm{HE}}^{1-\text { loop }}}{\partial \bar{F}^{\alpha}{ }_{\rho} \partial \bar{F}^{\beta}{ }_{\sigma} \partial \bar{F}^{\mu}{ }_{\gamma}}(x) \frac{\partial \mathcal{L}_{\mathrm{HE}}^{1-\text { loop }}}{\partial \bar{F}^{\gamma \nu}}\left(x^{\prime}\right) .
\end{aligned}
$$

Finally, we turn to the disconnected diagrams in figure 6 . The first three diagrams in figure $6(\mathrm{c})$ correspond to $i j_{\text {1-loop }}^{\rho}(p) j_{1 \text {-loop }}^{\sigma}\left(p^{\prime}\right), \quad i j_{1 \text {-loop }}^{\rho}(p) j_{2 \text {-loop }}^{\sigma}\left(p^{\prime}\right)$ and $i j_{2 \text {-loop }}^{\rho}(p) j_{1 \text {-loop }}^{\sigma}\left(p^{\prime}\right)$, where

$$
i j_{l \text {-loop }}^{\rho}(p) j_{l^{\prime} \text {-loop }}^{\sigma}\left(p^{\prime}\right)=-\left.\left.4 i p^{\alpha} p^{\prime \beta} \int_{x} e^{i x p} \frac{\partial \mathcal{L}_{\mathrm{HE}}^{l \text {-loop }}}{\partial \bar{F}_{\rho}^{\alpha}}(x)\right|_{1 \mathrm{PI}} \int_{x^{\prime}} e^{i x^{\prime} p^{\prime}} \frac{\partial \mathcal{L}_{\mathrm{HE}}^{l^{\prime} \text {-loop }}}{\partial \bar{F}_{\sigma}^{\beta}}\left(x^{\prime}\right)\right|_{1 \mathrm{PI}} .
$$

The two diagrams depicted in the last line of figure 6(c) amount to current-current correlators with one-loop quantum correction to the out- and ingoing photon line, respectively. The left one can be expressed as

$$
\begin{aligned}
& i j_{l \text {-loop }}^{\rho}(p) \int_{k} j_{1 \text {-loop }}^{\mu}(k) D_{\mu \nu}(k) \Pi_{1 \text {-loop }}^{\nu \sigma}\left(-k, p^{\prime}\right) \\
& \quad=4 i p^{\alpha} p^{\prime \beta} \int_{x^{\prime \prime}} e^{i x^{\prime \prime} p} \frac{\partial \mathcal{L}_{1-\text { loop }}}{\partial \bar{F}^{\alpha}{ }_{\rho}}\left(x^{\prime \prime}\right) \int_{x} \int_{x^{\prime}} e^{i x^{\prime} p^{\prime}} G^{\mu \nu}\left(x-x^{\prime}\right) \frac{\partial \mathcal{L}_{\text {HE }}^{1-\text { loop }}}{\partial \bar{F}_{\gamma}^{\mu}}(x) \frac{\partial^{2} \mathcal{L}_{\text {HE }}^{1-\text { loop }}}{\partial \bar{F}^{\gamma \nu} \partial \bar{F}_{\sigma}^{\beta}}\left(x^{\prime}\right),
\end{aligned}
$$

and the right one corresponds to eq. (4.20) with the replacements $p \leftrightarrow p^{\prime}$ and $\rho \leftrightarrow \sigma$.

In constant electromagnetic fields, all diagrams depicted in figure $6(\mathrm{c})$ vanish if at least one of the external photons is real; cf. the discussion in section 4.3. Of course, the derivatives of the Lagrangian for $\bar{F}$ in eqs. (4.17)-(4.20) could again be expressed in terms of derivatives for $\mathcal{F}$ and $\mathcal{G}$ (cf. appendix A). While this would allow us to identify the various tensor structures spanning these contributions, the resulting expressions are lengthy so that we do not show them here.

Correspondingly, the photon polarization tensor in the presence of an external field is not only given by 1PI diagrams, but also receives corrections from 1PR and even disconnected diagrams. More precisely, it is made up of all the diagrams that do not correspond to iterations of more elementary diagrams describing quantum corrections to photon propagation. The full dressed photon propagator in the external field - accounting for quantum corrections to all orders - is then obtained by summing up all possible iterations analogously to a Dyson series of this photon polarization tensor. At $l$ loops we have

$$
\Pi_{l \text {-loop }}^{\rho \sigma}\left(p, p^{\prime}\right)=\left.\Pi_{l \text {-loop }}^{\rho \sigma}\left(p, p^{\prime}\right)\right|_{1 \mathrm{PI}}+\Delta \Pi_{l \text {-loop }}^{\rho \sigma}\left(p, p^{\prime}\right),
$$

where $\left.\Pi_{l \text {-loop }}^{\rho \sigma}\right|_{1 \mathrm{PI}}:=\left.s_{(2)}^{\rho \sigma}\right|_{l \text {-loop }}$ and $\Delta \Pi_{2 \text {-loop }}^{\rho \sigma}$ refers to the contributions of $1 \mathrm{PR}$ and disconnected diagrams. In a slight abuse of nomenclature, we label contributions to the photon polarization tensor which scale as $\sim\left(\frac{\alpha}{\pi}\right)^{l}$ with "l-loop", even though $\Delta \Pi_{l \text {-loop }}^{\rho \sigma}$ generically also includes current-current correlators involving higher loop numbers; cf. figure 6 . The explicit expressions for $\Delta \Pi_{l \text {-loop }}^{\rho \sigma}$ at one and two loops are

$$
\Delta \Pi_{1 \text {-loop }}^{\rho \sigma}\left(p, p^{\prime}\right):=i j_{\text {1-loop }}^{\rho}(p) j_{1 \text {-loop }}^{\sigma}\left(p^{\prime}\right),
$$


and

$$
\begin{aligned}
\Delta \Pi_{2 \text {-loop }}^{\rho \sigma}\left(p, p^{\prime}\right):=2 \int_{k} s_{(3)}^{\rho \sigma \mu}\left(p, p^{\prime},-k\right) & \left.\right|_{1 \text {-loop }} D_{\mu \nu}(k) j_{1 \text {-loop }}^{\nu}(k) \\
& +i j_{\text {1-loop }}^{\rho}(p) j_{2 \text {-loop }}^{\sigma}\left(p^{\prime}\right)+i j_{2 \text {-loop }}^{\rho}(p) j_{1 \text {-loop }}^{\sigma}\left(p^{\prime}\right) .
\end{aligned}
$$

Equations (4.22) and (4.23) account for the five distinct diagrams in figure 6(b) and 6(c) that do not correspond to iterations of more elementary quantum corrections to photon propagation.

In inhomogeneous electromagnetic fields, the explicit expressions for eqs. (4.22) and (4.23) written in terms of derivatives of $\mathcal{L}_{\mathrm{HE}}^{\text {1-loop }}$ for the scalar field invariants $\mathcal{F}$ and $\mathcal{G}$ are rather lengthy. The main reasons for this are the different space-time arguments of the derivatives of $\mathcal{L}_{\mathrm{HE}}^{1-\text { loop }}$. However, in constant external fields these expressions simplify significantly, as all the current-current correlators vanish and the derivatives of $\mathcal{L}_{\mathrm{HE}}^{\text {1-loop }}$ with respect to $\bar{F}$ become independent of $x$. More specifically, in this limit, we obtain $\Delta \Pi_{1-\text { loop }}^{\rho \sigma}\left(p, p^{\prime}\right)=0$, and

$$
\begin{aligned}
\Delta \Pi_{2 \text {-loop }}^{\rho \sigma}\left(p, p^{\prime}\right) & =-(2 \pi)^{4} \delta\left(p+p^{\prime}\right) 8 p^{\mu_{1}} p^{\mu_{2}} \frac{\partial^{3} \mathcal{L}_{\mathrm{HE}}^{1 \text {-loo }}}{\partial \bar{F}^{\mu_{1}} \partial \bar{F}^{\mu_{2}}{ }_{\sigma} \partial \bar{F}^{\mu \nu_{3}}} \frac{\partial \mathcal{L}_{\mathrm{HE}}^{\text {1-loop }}}{\partial \bar{F}_{\nu_{3} \mu}} \\
& =(2 \pi)^{4} \delta\left(p+p^{\prime}\right) \sum_{j=0}^{3} P_{j}^{\rho \sigma}(p) \Delta c_{j}^{2 \text {-loop }}(\mathcal{F}, \mathcal{G}),
\end{aligned}
$$

with the tensor structures $P_{j}^{\rho \sigma}(p)$ defined in eq. (4.15). Hence, eq. (4.24) is spanned by the same tensor structures as $\left.\Pi^{\rho \sigma}\right|_{1 \mathrm{PI}}$ in constant fields as listed in eq. (4.15). The coefficients $\Delta c_{j}^{2 \text {-loop }}$ are given by

$$
\begin{aligned}
\Delta c_{0}^{2 \text {-loop }} & =\left(\bar{F} \partial_{\bar{F}} \mathcal{L}\right) \frac{\partial^{2} \mathcal{L}}{\partial \mathcal{F}^{2}}+\left({ }^{*} \bar{F} \partial_{\bar{F}} \mathcal{L}\right) \frac{\partial^{2} \mathcal{L}}{\partial \mathcal{F} \partial \mathcal{G}}, \\
\Delta c_{1}^{2 \text {-loop }} & =\left(\bar{F} \partial_{\bar{F}} \mathcal{L}\right) \frac{\partial^{3} \mathcal{L}}{\partial \mathcal{F}^{3}}+\left({ }^{*} \bar{F} \partial_{\bar{F}} \mathcal{L}\right) \frac{\partial^{3} \mathcal{L}}{\partial \mathcal{F}^{2} \partial \mathcal{G}}+2 \frac{\partial \mathcal{L}}{\partial \mathcal{F}} \frac{\partial^{2} \mathcal{L}}{\partial \mathcal{F}^{2}}-2 \frac{\partial \mathcal{L}}{\partial \mathcal{G}} \frac{\partial^{2} \mathcal{L}}{\partial \mathcal{F} \partial \mathcal{G}}, \\
\Delta c_{2}^{2 \text {-loop }} & =\left(\bar{F} \partial_{\bar{F}} \mathcal{L}\right) \frac{\partial^{3} \mathcal{L}}{\partial \mathcal{F} \partial \mathcal{G}^{2}}+\left({ }^{*} \bar{F} \partial_{\bar{F}} \mathcal{L}\right) \frac{\partial^{3} \mathcal{L}}{\partial \mathcal{G}^{3}}+2 \frac{\partial \mathcal{L}}{\partial \mathcal{F}} \frac{\partial^{2} \mathcal{L}}{\partial \mathcal{G}^{2}}+2 \frac{\partial \mathcal{L}}{\partial \mathcal{G}} \frac{\partial^{2} \mathcal{L}}{\partial \mathcal{F} \partial \mathcal{G}}, \\
\Delta c_{3}^{2 \text {-loop }} & =\left(\bar{F} \partial_{\bar{F}} \mathcal{L}\right) \frac{\partial^{3} \mathcal{L}}{\partial \mathcal{F}^{2} \partial \mathcal{G}}+\left({ }^{*} \bar{F} \partial_{\bar{F}} \mathcal{L}\right) \frac{\partial^{3} \mathcal{L}}{\partial \mathcal{F} \partial \mathcal{G}^{2}}+2 \frac{\partial \mathcal{L}}{\partial \mathcal{F}} \frac{\partial^{2} \mathcal{L}}{\partial \mathcal{F} \partial \mathcal{G}}+\frac{\partial \mathcal{L}}{\partial \mathcal{G}}\left(\frac{\partial^{2} \mathcal{L}}{\partial \mathcal{F}^{2}}-\frac{\partial^{2} \mathcal{L}}{\partial \mathcal{G}^{2}}\right),
\end{aligned}
$$

where $\mathcal{L}=\mathcal{L}_{\mathrm{HE}}^{1 \text {-loop }}$, and we have made use of the shorthand notations $\left(\bar{F} \partial_{\bar{F}} \mathcal{L}\right):=\bar{F}^{\mu \nu} \frac{\partial \mathcal{L}}{\partial \bar{F}^{\mu \nu}}=$ $2\left(\mathcal{F} \frac{\partial \mathcal{L}}{\partial \mathcal{F}}+\mathcal{G} \frac{\partial \mathcal{L}}{\partial \mathcal{G}}\right)$ and $\left({ }^{*} \bar{F} \partial_{\bar{F}} \mathcal{L}\right):={ }^{*} \bar{F}^{\mu \nu} \frac{\partial \mathcal{L}}{\partial \bar{F}^{\mu \nu}}=2\left(\mathcal{G} \frac{\partial \mathcal{L}}{\partial \mathcal{F}}-\mathcal{F} \frac{\partial \mathcal{L}}{\partial \mathcal{G}}\right)$.

It is instructive to compare the coefficients $\Delta c_{j}^{2 \text {-loop }}$ in eq. (4.25) with the coefficients $c_{j}^{2 \text {-loop }}$ in eq. (4.16). This comparison is rather straightforward in the limits of weak and strong fields. For spinor QED, the weak-field expressions for the coefficients (4.16) and (4.25) can be obtained with the help of eqs. (3.3) and (3.4). They are given by

$$
\begin{aligned}
c_{0}^{2 \text {-loop }} & =\left(\frac{\alpha}{\pi}\right)^{2}\left[\frac{32}{81} \tilde{\mathcal{F}}-\frac{1}{45^{2}}\left(\frac{3657}{2} \tilde{\mathcal{F}}^{2}+1082 \tilde{\mathcal{G}}^{2}\right)+\mathcal{O}\left(\epsilon^{6}\right)\right], \\
c_{1}^{2 \text {-loop }} & =\frac{e^{2}}{m^{4}}\left(\frac{\alpha}{\pi}\right)^{2}\left[\frac{32}{81}-\frac{3657}{45^{2}} \tilde{\mathcal{F}}+\mathcal{O}\left(\epsilon^{4}\right)\right],
\end{aligned}
$$




$$
\begin{aligned}
c_{2}^{2 \text {-loop }} & =\frac{e^{2}}{m^{4}}\left(\frac{\alpha}{\pi}\right)^{2}\left[\frac{263}{324}-\frac{2164}{45^{2}} \tilde{\mathcal{F}}+\mathcal{O}\left(\epsilon^{4}\right)\right], \\
c_{3}^{2 \text {-loop }} & =-\frac{e^{2}}{m^{4}}\left(\frac{\alpha}{\pi}\right)^{2}\left[\frac{2164}{45^{2}} \tilde{\mathcal{G}}+\mathcal{O}\left(\epsilon^{4}\right)\right],
\end{aligned}
$$

and

$$
\begin{aligned}
& \Delta c_{0}^{2 \text {-loop }}=\left(\frac{\alpha}{\pi}\right)^{2}\left[\frac{1}{45^{2}}\left(32 \tilde{\mathcal{F}}^{2}+56 \tilde{\mathcal{G}}^{2}\right)+\mathcal{O}\left(\epsilon^{6}\right)\right], \\
& \Delta c_{1}^{2 \text {-loop }}=\frac{e^{2}}{m^{4}}\left(\frac{\alpha}{\pi}\right)^{2}\left[\frac{32}{45^{2}} \tilde{\mathcal{F}}+\mathcal{O}\left(\epsilon^{4}\right)\right] \\
& \Delta c_{2}^{2 \text {-loop }}=\frac{e^{2}}{m^{4}}\left(\frac{\alpha}{\pi}\right)^{2}\left[\frac{56}{45^{2}} \tilde{\mathcal{F}}+\mathcal{O}\left(\epsilon^{4}\right)\right] \\
& \Delta c_{3}^{2 \text {-loop }}=-\frac{e^{2}}{m^{4}}\left(\frac{\alpha}{\pi}\right)^{2}\left[\frac{21}{45^{2}} \tilde{\mathcal{G}}+\mathcal{O}\left(\epsilon^{4}\right)\right] .
\end{aligned}
$$

We infer that these coefficients fulfill $\Delta c_{j}^{2 \text {-loop }} / c_{j}^{2 \text {-loop }}=\mathcal{O}\left(\epsilon^{2}\right)$ for $j \in\{0,1,2\}$, and $\Delta c_{3}^{2 \text {-loop }} / c_{3}^{2 \text {-loop }}=\mathcal{O}(1)$. For $|\tilde{\mathcal{F}}| \gg 1$ and $|\tilde{\mathcal{G}}| \ll 1$, the coefficients $c_{j}^{2 \text {-loop }}$ follow from eq. (3.7) by differentiation and read

$$
\begin{aligned}
c_{0}^{2 \text {-loop }} & =\left(\frac{\alpha}{\pi}\right)^{2}\left[\frac{1}{4} \ln \sqrt{\tilde{\mathcal{F}}}+\mathcal{O}\left(\left(\frac{1}{\tilde{\mathcal{F}}}\right)^{0}\right)+\mathcal{O}\left(\tilde{\mathcal{G}}^{2}\right)\right], \\
c_{1}^{2 \text {-loop }} & =\frac{1}{\mathcal{F}}\left(\frac{\alpha}{\pi}\right)^{2}\left[\frac{1}{8}+\mathcal{O}(1 / \sqrt{\tilde{\mathcal{F}}})+\mathcal{O}\left(\tilde{\mathcal{G}}^{2}\right)\right], \\
c_{2}^{2 \text {-loop }} & =\frac{1}{\mathcal{F}}\left(\frac{\alpha}{\pi}\right)^{2} \sqrt{\tilde{\mathcal{F}}}\left[-\frac{1}{6 \sqrt{2}}+\mathcal{O}(1 / \sqrt{\tilde{\mathcal{F}}})+\mathcal{O}\left(\tilde{\mathcal{G}}^{2}\right)\right], \\
c_{3}^{2 \text {-loop }} & =\frac{1}{\mathcal{F}}\left(\frac{\alpha}{\pi}\right)^{2} \frac{\tilde{\mathcal{G}}}{\sqrt{\tilde{\mathcal{F}}}}\left[\frac{1}{12 \sqrt{2}}+\mathcal{O}(1 / \sqrt{\tilde{\mathcal{F}}})+\mathcal{O}\left(\tilde{\mathcal{G}}^{2}\right)\right] .
\end{aligned}
$$

The analogous coefficients $\Delta c_{j}^{2 \text {-loop }}$ can be obtained from eq. (4.25) by using the expressions assembled in eq. (B.6), yielding

$$
\begin{aligned}
\Delta c_{0}^{2 \text {-loop }} & =\left(\frac{\alpha}{\pi}\right)^{2}\left[\frac{1}{9} \ln \sqrt{\tilde{\mathcal{F}}}+\mathcal{O}\left(\left(\frac{1}{\tilde{\mathcal{F}}}\right)^{0}\right)+\mathcal{O}\left(\tilde{\mathcal{G}}^{2}\right)\right], \\
\Delta c_{1}^{2 \text {-loop }} & =\frac{1}{\mathcal{F}}\left(\frac{\alpha}{\pi}\right)^{2}\left[\mathcal{O}(1 / \sqrt{\tilde{\mathcal{F}}})+\mathcal{O}\left(\tilde{\mathcal{G}}^{2}\right)\right], \\
\Delta c_{2}^{2 \text {-loop }} & =\frac{1}{\mathcal{F}}\left(\frac{\alpha}{\pi}\right)^{2} \sqrt{\tilde{\mathcal{F}}}\left[\frac{1}{9 \sqrt{2}} \ln \sqrt{\tilde{\mathcal{F}}}+\mathcal{O}\left(\left(\frac{1}{\tilde{\mathcal{F}}}\right)^{0}\right)+\mathcal{O}\left(\tilde{\mathcal{G}}^{2}\right)\right], \\
\Delta c_{3}^{2 \text {-loop }} & =\frac{1}{\mathcal{F}}\left(\frac{\alpha}{\pi}\right)^{2} \frac{\tilde{\mathcal{G}}}{\sqrt{\tilde{\mathcal{F}}}}\left[-\frac{1}{18 \sqrt{2}} \ln \sqrt{\tilde{\mathcal{F}}}+\mathcal{O}\left(\left(\frac{1}{\tilde{\mathcal{F}}}\right)^{0}\right)+\mathcal{O}\left(\tilde{\mathcal{G}}^{2}\right)\right] .
\end{aligned}
$$

Hence, in the strong-field limit, we read off the scalings $\Delta c_{0}^{2 \text {-loop }} / c_{0}^{2 \text {-loop }}=\mathcal{O}(1)$, $\Delta c_{1}^{2 \text {-loop }} / c_{1}^{2 \text {-loop }}=\mathcal{O}(1 / \sqrt{\tilde{\mathcal{F}}})$ and $\Delta c_{2}^{2 \text {-loop }} / c_{2}^{2 \text {-loop }} \sim \Delta c_{3}^{2 \text {-loop }} / c_{3}^{2 \text {-loop }} \sim-\frac{2}{3} \ln \sqrt{\tilde{\mathcal{F}}}$. This implies that the contribution of $\Delta c_{1}^{2 \text {-loop }}$ is always suppressed in comparison to $c_{1}^{2 \text {-loop }}$ 
for large values of $|\tilde{\mathcal{F}}| \gg 1$. By contrast, $\Delta c_{j}^{2 \text {-loop }}$ can surpass $c_{j}^{2 \text {-loop }}$ in magnitude for $j \in\{2,3\}$. Physically, the $c_{2}$ coefficient is responsible for the enhanced refractive properties of low-frequency photons in a strong field, affecting, e.g., the mode polarized in the plane spanned by a magnetic field and the propagation direction in a magnetized quantum vacuum. For completeness, also note that $\Delta c_{2}^{2 \text {-loop }} / c_{2}^{1 \text {-loop }} \sim \Delta c_{3}^{2 \text {-loop }} / c_{3}^{1-\text { loop }} \sim \frac{1}{3} \frac{\alpha}{\pi} \ln \sqrt{\tilde{\mathcal{F}}}$; cf. the discussion of the analogous considerations for $\mathcal{L}_{\mathrm{HE}}^{\text {2-loops }}$ in constant fields in section 3.

\section{Conclusions and outlook}

We have taken a fresh look at the famous Heisenberg-Euler effective action $\Gamma_{\mathrm{HE}}$, which has played a substantial role in the development of quantum field theory, and its relation to the underlying microscopic theory of QED formulated in terms of the partion function. We have paid particular attention to the differences and common ground of $\Gamma_{\mathrm{HE}}$ and the nowadays more conventional 1PI effective action. Most noteworthily and distinctively, $\Gamma_{\mathrm{HE}}$ also contains $1 \mathrm{PR}$ contributions implying quantitative differences to the 1PI effective action from the two-loop level on.

For an efficient determination of these effective actions, we have constructed an effective theory of low-frequency photons in the QED vacuum subject to slowly varying electromagnetic fields. Apart from discussing various generic features of such an effective theory, our main focus was on the effective interactions generated at $\mathcal{O}\left(\left(\frac{\alpha}{\pi}\right)^{2}\right)$. Here, we in particular studied the effective self-interaction of external electromagnetic fields, and derived the photon polarization tensor in the low-frequency limit. The latter is composed of 1PI, 1PR as well as disconnected diagrams.

One of our main findings is that the coupling of two one-loop vacuum currents via a photon propagator gives rise to a nonvanishing $1 \mathrm{PR}$ contribution to $\Gamma_{\mathrm{HE}}^{2 \text {-loop }}$ even in the limit of constant electromagnetic fields; cf. figure 4 (right). This contribution was previously believed to vanish. To clarify the importance of this newly evaluated 1PR contribution relatively to the well-known 1PI one, we have investigated the limits of perturbatively weak and strong fields. Whereas $\left.\Gamma_{\mathrm{HE}}^{2 \text {-loop }}\right|_{1 \mathrm{PR}}$ is generically suppressed for weak fields, it can even surpass $\left.\Gamma_{\mathrm{HE}}^{2 \text {-loop }}\right|_{1 \mathrm{PI}}$ in magnitude for strong fields. Similar results are obtained for the two-loop photon polarization tensor. Also here, the 1PR contributions can surpass the 1PI ones in magnitude for strong fields.

Our results can also be of relevance beyond QED, for instance, for the exotic case of a hypothetical minicharged particle sector beyond the Standard Model of particle physics [87]. Beyond QED, the most essential new feature of the diagram depicted in figure 4 (right) as compared to figure 4 (left) is that the first one can induce effective interactions mediated by two different fermion species (the two loops in this diagram do not necessarily have to contain the same fermion species), while the latter one features a single fermion loop and thus may only involve one fermion species. This implies a parametrically different dependence of electromagnetic or optical observables on the various coupling and mass parameters starting at two-loop level. As the considerations invoked here can also be adopted for scalar QED, particularly in a combination of scalar and spinor QED, this type of mixed effective interactions can also be generated when one of the loops traces over 
fermions and the other one over bosons. For 1PI diagrams, such an effective coupling of different species can only happen at three loops or beyond.

\section{Acknowledgments}

We acknowledge support by the BMBF under grant No. 05P15SJFAA (FAIR-APPASPARC). The authors wish to thank W. Dittrich for helpful conversations and for carefully reading the manuscript.

\section{A Useful derivative identities}

The Heisenberg-Euler effective Lagrangian can be expressed as $\mathcal{L}_{\mathrm{HE}}=\sum_{l=0}^{\infty} \mathcal{L}_{\mathrm{HE}}^{l \text {-loop }}$, where $\mathcal{L}_{\mathrm{HE}}^{0 \text {-loop }}=-\mathcal{F}$ is the Maxwell Lagrangian of classical electrodynamics, and $\mathcal{L}_{\mathrm{HE}}^{l \text {-loop }}$ with $l \geq 1$ encodes quantum corrections vanishing in the formal limit $\hbar \rightarrow 0$. In constant fields, we have $\mathcal{L}_{\mathrm{HE}}^{\text {l-loop }} \equiv \mathcal{L}_{\mathrm{HE}}^{l \text {-loop }}\left(\mathcal{F}, \mathcal{G}^{2}\right)$; cf. the main text for more details. As $\mathcal{L}_{\mathrm{HE}}^{l \text {-loop }}$ is a function of the scalar invariants of the electromagnetic field $\mathcal{F}$ and $\mathcal{G}^{2}$ only, it is convenient to trade derivatives with respect to the field strength tensor $\bar{F}$ for derivatives with respect to $\mathcal{F}$ and $\mathcal{G}$. The explicit expressions for these transformations up to cubic order in the derivative for $\bar{F}$ are

$$
\begin{aligned}
\frac{\partial \mathcal{L}_{\mathrm{HE}}^{l \text {-loop }}}{\partial \bar{F}^{\mu \nu}}= & \frac{1}{2}\left(\bar{F}_{\mu \nu} \frac{\partial}{\partial \mathcal{F}}+{ }^{*} \bar{F}_{\mu \nu} \frac{\partial}{\partial \mathcal{G}}\right) \mathcal{L}_{\mathrm{HE}}^{l \text {-loop }} \\
\frac{\partial^{2} \mathcal{L}_{\mathrm{HE}}^{l \text { lloop }}}{\partial \bar{F}^{\alpha \beta} \partial \bar{F}^{\mu \nu}}= & \frac{1}{4}\left[\left(g_{\alpha \mu} g_{\beta \nu}-g_{\alpha \nu} g_{\beta \mu}\right) \frac{\partial}{\partial \mathcal{F}}+\epsilon_{\mu \nu \alpha \beta} \frac{\partial}{\partial \mathcal{G}}+\bar{F}_{\alpha \beta} \bar{F}_{\mu \nu} \frac{\partial^{2}}{\partial \mathcal{F}^{2}}+{ }^{*} \bar{F}_{\alpha \beta}{ }^{*} \bar{F}_{\mu \nu} \frac{\partial^{2}}{\partial \mathcal{G}^{2}}\right. \\
& \left.+\left({ }^{*} \bar{F}_{\alpha \beta} \bar{F}_{\mu \nu}+\bar{F}_{\alpha \beta}{ }^{*} \bar{F}_{\mu \nu}\right) \frac{\partial^{2}}{\partial \mathcal{F} \partial \mathcal{G}}\right] \mathcal{L}_{\mathrm{HE}}^{l \text {-loop }}
\end{aligned}
$$

and

$$
\begin{aligned}
\frac{\partial^{3} \mathcal{L}_{\mathrm{HE}}^{l \text {-loop }}}{\partial \bar{F}^{\rho \sigma} \partial \bar{F}^{\alpha \beta} \partial \bar{F}^{\mu \nu}}=\frac{1}{8}\{ & \bar{F}_{\rho \sigma} \bar{F}_{\alpha \beta} \bar{F}_{\mu \nu} \frac{\partial^{3}}{\partial \mathcal{F}^{3}}+{ }^{*} \bar{F}_{\rho \sigma}{ }^{*} \bar{F}_{\alpha \beta}{ }^{*} \bar{F}_{\mu \nu} \frac{\partial^{3}}{\partial \mathcal{G}^{3}} \\
& +\left(\bar{F}_{\rho \sigma}{ }^{*} \bar{F}_{\alpha \beta} \bar{F}_{\mu \nu}+\bar{F}_{\rho \sigma} \bar{F}_{\alpha \beta}{ }^{*} \bar{F}_{\mu \nu}+{ }^{*} \bar{F}_{\rho \sigma} \bar{F}_{\alpha \beta} \bar{F}_{\mu \nu}\right) \frac{\partial^{3}}{\partial \mathcal{F}^{2} \partial \mathcal{G}} \\
& +\left({ }^{*} \bar{F}_{\rho \sigma}{ }^{*} \bar{F}_{\alpha \beta} \bar{F}_{\mu \nu}+{ }^{*} \bar{F}_{\rho \sigma} \bar{F}_{\alpha \beta}{ }^{*} \bar{F}_{\mu \nu}+\bar{F}_{\rho \sigma}{ }^{*} \bar{F}_{\alpha \beta}{ }^{*} \bar{F}_{\mu \nu}\right) \frac{\partial^{3}}{\partial \mathcal{F} \partial \mathcal{G}^{2}} \\
& +\left[\left(g_{\alpha \mu} g_{\beta \nu}-g_{\alpha \nu} g_{\beta \mu}\right) \bar{F}_{\rho \sigma}+\left(g_{\rho \mu} g_{\sigma \nu}-g_{\rho \nu} g_{\sigma \mu}\right) \bar{F}_{\alpha \beta}\right. \\
& \left.+\left(g_{\rho \alpha} g_{\sigma \beta}-g_{\rho \beta} g_{\sigma \alpha}\right) \bar{F}_{\mu \nu}\right] \frac{\partial^{2}}{\partial \mathcal{F}^{2}} \\
& +\left(\epsilon_{\mu \nu \alpha \beta}{ }^{*} \bar{F}_{\rho \sigma}+\epsilon_{\mu \nu \rho \sigma}{ }^{*} \bar{F}_{\alpha \beta}+\epsilon_{\alpha \beta \rho \sigma}{ }^{*} \bar{F}_{\mu \nu}\right) \frac{\partial^{2}}{\partial \mathcal{G}^{2}} \\
& +\left[\left(\epsilon_{\mu \nu \alpha \beta} \bar{F}_{\rho \sigma}+\epsilon_{\mu \nu \rho \sigma} \bar{F}_{\alpha \beta}+\epsilon_{\alpha \beta \rho \sigma} \bar{F}_{\mu \nu}\right)\right.
\end{aligned}
$$




$$
\begin{aligned}
& +\left(g_{\alpha \mu} g_{\beta \nu}-g_{\alpha \nu} g_{\beta \mu}\right)^{*} \bar{F}_{\rho \sigma}+\left(g_{\rho \mu} g_{\sigma \nu}-g_{\rho \nu} g_{\sigma \mu}\right)^{*} \bar{F}_{\alpha \beta} \\
& \left.\left.+\left(g_{\rho \alpha} g_{\sigma \beta}-g_{\rho \beta} g_{\sigma \alpha}\right)^{*} \bar{F}_{\mu \nu}\right] \frac{\partial^{2}}{\partial \mathcal{F} \partial \mathcal{G}}\right\} \mathcal{L}_{\mathrm{HE}}^{l \text { loop }}
\end{aligned}
$$

\section{B Strong-field asymptotics for spinor QED}

In this appendix we concentrate on constant fields and focus on the limit of $|\tilde{\mathcal{F}}|=\frac{1}{2} \mid\left(\frac{e \vec{B}}{m^{2}}\right)^{2}-$ $\left(\frac{e \vec{E}}{m^{2}}\right)^{2} \mid \gg 1$ and $|\tilde{\mathcal{G}}|=\left|\frac{e \vec{E}}{m^{2}} \cdot \frac{e \vec{B}}{m^{2}}\right| \ll 1$, which is of relevance for either strong electric or magnetic fields.

\section{B.1 One-loop Heisenberg-Euler effective Lagrangian in constant external fields}

For the special case of $\mathcal{G}=0$, the one-loop Heisenberg-Euler effective Lagrangian has the following closed-form representation [4, 71, 88],

$$
\left.\frac{\mathcal{L}_{\mathrm{HE}}^{1 \text {-loop }}}{m^{4}}\right|_{\mathcal{G}=0}=\frac{1}{4 \pi^{2}} \frac{1}{2 \chi^{2}}\left\{\zeta^{\prime}(-1, \chi)+\frac{\chi}{2}\left[(1-\chi) \ln \chi+\frac{\chi}{2}\right]-\frac{1}{12}(\ln \chi+1)\right\},
$$

where $\chi=\frac{1}{2} / \sqrt{2 \tilde{\mathcal{F}}}$ and $\zeta^{\prime}(s, \chi)=\partial_{s} \zeta(s, \chi)$ denotes the first derivative of the Hurwitz zeta function; cf. eq. (3.8) for the definition of the square root of $\tilde{\mathcal{F}}$. In principle, similar closedform expressions can be obtained for any derivative $\left.\frac{\partial^{n_{1}+n_{2}} \mathcal{L}_{\mathrm{HE}}^{1 \text {-loop }}}{\partial \mathcal{F}^{n} \partial \mathcal{G}^{n}}\right|_{\mathcal{G}=0}$ with $\left\{n_{1}, n_{2}\right\} \in \mathbb{N}_{0}$. Here we only provide the following explicit expressions [68],

$$
\begin{aligned}
& \left.\frac{\partial \mathcal{L}_{\mathrm{HE}}^{1 \text {-loop }}}{\partial \mathcal{F}}\right|_{\mathcal{G}=0}=\frac{\alpha}{\pi}\left\{4 \zeta^{\prime}(-1, \chi)-\chi\left[2 \zeta^{\prime}(0, \chi)-\ln \chi+\chi\right]-\frac{1}{3} \ln \chi-\frac{1}{2}\right\}, \\
& \left.\frac{\partial^{2} \mathcal{L}_{\mathrm{HE}}^{1-\text { loop }}}{\partial \mathcal{G}^{2}}\right|_{\mathcal{G}=0}=\frac{1}{2 \mathcal{F}} \frac{\alpha}{\pi}\left\{4 \zeta^{\prime}(-1, \chi)-\chi\left[2 \zeta^{\prime}(0, \chi)-\ln \chi+\chi\right]-\frac{1}{6}\left[2 \psi(\chi)+\frac{1}{\chi}+1\right]\right\},
\end{aligned}
$$

where $\psi(\chi)=\frac{\mathrm{d}}{\mathrm{d} \chi} \ln \Gamma(\chi)$ is the Digamma function. Obviously, we can write

$$
\frac{\mathcal{L}_{\mathrm{HE}}^{1 \text {-loop }}}{m^{4}}=\left.\frac{\mathcal{L}_{\mathrm{HE}}^{1 \text {-loop }}}{m^{4}}\right|_{\mathcal{G}=0}+\left.\frac{1}{2 m^{4}} \frac{\partial^{2} \mathcal{L}_{\mathrm{HE}}^{1 \text {-loop }}}{\partial \mathcal{G}^{2}}\right|_{\mathcal{G}=0} \mathcal{G}^{2}+\mathcal{O}\left(\mathcal{G}^{4}\right)
$$

such that, upon insertion of eqs. (B.1) and (B.2), eq. (B.3) provides us with a closed-form expression of $\mathcal{L}_{\mathrm{HE}}^{1-\text { loop }} / m^{4}$ in the limit of $|\tilde{\mathcal{G}}| \ll 1$. Aiming at extracting the asymptotics of eq. (B.3) for $|\tilde{\mathcal{F}}| \gg 1 \leftrightarrow|\chi| \ll 1$, it is convenient to employ [89]

$$
\zeta^{\prime}(0, \chi)=\ln \Gamma(\chi)-\frac{1}{2} \ln (2 \pi)
$$

and eq. (1.50) of [71] (cf. also appendix D.6 of [5]),

$$
\zeta^{\prime}(-1, \chi)=\int_{0}^{\chi} \mathrm{dt} \ln \Gamma(\mathrm{t})+\frac{\chi}{2}(\chi-1)-\frac{\chi}{2} \ln (2 \pi)+\zeta^{\prime}(-1),
$$

where $\Gamma($.$) is the Gamma function, and \zeta^{\prime}(-1) \approx-0.16542114$ is the first derivative of the Riemann zeta function evaluated at -1 . The leading terms of an expansion of $\ln \Gamma($. for small arguments can then be inferred from eq. (1.53) [71]. As a result, we obtain 
eq. (3.6) in the main text. The asymptotics of the various derivatives of $\mathcal{L}_{\mathrm{HE}}^{1 \text {-loop }}$ for $\mathcal{F}$ and $\mathcal{G}$ determining $\left.\mathcal{L}_{\mathrm{HE}}^{2 \text {-loop }}\right|_{1 \mathrm{PR}}, c_{1 \text {-loop }}^{(n)}$ and $\Delta c_{2 \text {-loop }}^{(n)}$ can be inferred along the same lines and read

$$
\begin{aligned}
& \frac{\partial \mathcal{L}_{\mathrm{HE}}^{1 \text {-loop }}}{\partial \mathcal{F}}=\frac{\alpha}{\pi}\left[\frac{1}{3} \ln \sqrt{\tilde{\mathcal{F}}}+\mathcal{O}\left(\left(\frac{1}{\tilde{\mathcal{F}}}\right)^{0}\right)+\mathcal{O}\left(\tilde{\mathcal{G}}^{2}\right)\right], \\
& \frac{\partial \mathcal{L}_{\mathrm{HE}}^{1 \text {-loop }}}{\partial \mathcal{G}}=\frac{\alpha}{\pi} \frac{\tilde{\mathcal{G}}}{\sqrt{\tilde{\mathcal{F}}}}\left[\frac{1}{3 \sqrt{2}}+\mathcal{O}(1 / \sqrt{\tilde{\mathcal{F}}})+\mathcal{O}\left(\tilde{\mathcal{G}}^{2}\right)\right], \\
& \frac{\partial^{2} \mathcal{L}_{\mathrm{HE}}^{1 \text { lloop }}}{\partial \mathcal{F}^{2}}=\frac{1}{\mathcal{F}} \frac{\alpha}{\pi}\left[\frac{1}{6}+\mathcal{O}(1 / \sqrt{\tilde{\mathcal{F}}})+\mathcal{O}\left(\tilde{\mathcal{G}}^{2}\right)\right], \\
& \frac{\partial^{2} \mathcal{L}_{\text {HE }}^{\text {1-loop }}}{\partial \mathcal{G}^{2}}=\frac{1}{\mathcal{F}} \frac{\alpha}{\pi} \sqrt{\tilde{\mathcal{F}}}\left[\frac{1}{3 \sqrt{2}}+\mathcal{O}(1 / \sqrt{\tilde{\mathcal{F}}})+\mathcal{O}\left(\tilde{\mathcal{G}}^{2}\right)\right], \\
& \frac{\partial^{2} \mathcal{L}_{\text {HE }}^{1 \text {-loop }}}{\partial \mathcal{F} \partial \mathcal{G}}=\frac{1}{\mathcal{F}} \frac{\alpha}{\pi} \frac{\tilde{\mathcal{G}}}{\sqrt{\tilde{\mathcal{F}}}}\left[-\frac{1}{6 \sqrt{2}}+\mathcal{O}(1 / \sqrt{\tilde{\mathcal{F}}})+\mathcal{O}\left(\tilde{\mathcal{G}}^{2}\right)\right], \\
& \frac{\partial^{3} \mathcal{L}_{\mathrm{HE}}^{1 \text {-loop }}}{\partial \mathcal{F}^{3}}=\frac{1}{\mathcal{F}^{2}} \frac{\alpha}{\pi}\left[-\frac{1}{6}+\mathcal{O}(1 / \sqrt{\tilde{\mathcal{F}}})+\mathcal{O}\left(\tilde{\mathcal{G}}^{2}\right)\right], \\
& \frac{\partial^{3} \mathcal{L}_{\text {HE }}^{1 \text {-loop }}}{\partial \mathcal{F} \partial \mathcal{G}^{2}}=\frac{1}{\mathcal{F}^{2}} \frac{\alpha}{\pi} \sqrt{\tilde{\mathcal{F}}}\left[-\frac{1}{6 \sqrt{2}}+\mathcal{O}(1 / \sqrt{\tilde{\mathcal{F}}})+\mathcal{O}\left(\tilde{\mathcal{G}}^{2}\right)\right] \text {, } \\
& \frac{\partial^{3} \mathcal{L}_{\text {HE }}^{\text {1-loo }}}{\partial \mathcal{F}^{2} \partial \mathcal{G}}=\frac{1}{\mathcal{F}^{2}} \frac{\alpha}{\pi} \frac{\tilde{\mathcal{G}}}{\sqrt{\tilde{\mathcal{F}}}}\left[\frac{1}{4 \sqrt{2}}+\mathcal{O}(1 / \sqrt{\tilde{\mathcal{F}}})+\mathcal{O}\left(\tilde{\mathcal{G}}^{2}\right)\right], \\
& \frac{\partial^{3} \mathcal{L}_{\mathrm{HE}}^{1 \text {-loop }}}{\partial^{3} \mathcal{G}}=\frac{1}{\mathcal{F}^{2}} \frac{\alpha}{\pi} \frac{\tilde{\mathcal{G}}}{\sqrt{\tilde{\mathcal{F}}}}\left[\mathcal{O}\left(\left(\frac{1}{\tilde{\mathcal{F}}}\right)^{0}\right)+\mathcal{O}\left(\tilde{\mathcal{G}}^{2}\right)\right] .
\end{aligned}
$$

\section{B.2 Two-loop effective Lagrangian in constant external fields}

As detailed in the main text, the two-loop Heisenberg-Euler effective Lagrangian consists of a one-particle irreducible and a one-particle reducible contribution, $\mathcal{L}_{\mathrm{HE}}^{2 \text {-loo }}=\left.\mathcal{L}_{\mathrm{HE}}^{2 \text {-loop }}\right|_{1 \mathrm{PI}}+$ $\left.\mathcal{L}_{\mathrm{HE}}^{2 \text {-loop }}\right|_{1 \mathrm{PR}}$; cf. also figure 4 . The one-particle reducible contribution $\left.\mathcal{L}_{\mathrm{HE}}^{2 \text {-loop }}\right|_{1 \mathrm{PR}}$ follows straightforwardly from $\mathcal{L}_{\mathrm{HE}}^{\text {1-loop }}$ via eq. (3.2). For the special case of $\mathcal{G}=0$, it has the following closed-form representation:

$$
\left.\frac{\left.\mathcal{L}_{\mathrm{HE}}^{2 \text {-loo }}\right|_{1 \mathrm{PR}}}{m^{4}}\right|_{\mathcal{G}=0}=\frac{\alpha}{\pi} \frac{1}{4 \pi^{2}} \frac{1}{2 \chi^{2}}\left\{2 \zeta^{\prime}(-1, \chi)-\frac{\chi}{2}\left[2 \zeta^{\prime}(0, \chi)-\ln \chi+\chi\right]-\frac{1}{6}\left(\ln \chi+\frac{3}{2}\right)\right\}^{2} ;
$$

cf. also appendix B.1.

Closed-form expression exists for $\left.\mathcal{L}_{\mathrm{HE}}^{2 \text {-loop }}\right|_{1 \mathrm{PI}}$ only for the simplified case of self-dual fields [90], but not for the cases of interest here, not even for the special case of $\mathcal{G}=0$. However, the leading strong-field behavior of $\left.\mathcal{L}_{\mathrm{HE}}^{2 \text {-loop }}\right|_{1 \mathrm{PI}} / m^{4}$ for $|\tilde{\mathcal{F}}| \gg 1$ and $|\tilde{\mathcal{G}}| \ll 1$ is known explicitly [65] (cf. also [4] for an independent verification). For $\tilde{\mathcal{F}}>0$, it is given by

$$
\frac{\left.\mathcal{L}_{\mathrm{HE}}^{2 \text {-loop }}\right|_{1 \mathrm{PI}}}{m^{4}} \sim \frac{\alpha}{\pi} \frac{1}{4 \pi^{2}} \frac{\tilde{\eta}^{2}}{8}(\ln \tilde{\eta}+\text { constant })+\ldots
$$


where $\tilde{\eta}$ is one of the secular invariants of the electromagnetic field, defined as

$$
\tilde{\eta}=\left(\sqrt{\tilde{\mathcal{F}}^{2}+\tilde{\mathcal{G}}^{2}}+\tilde{\mathcal{F}}\right)^{1 / 2} \text { and } \quad \tilde{\epsilon}=\left(\sqrt{\tilde{\mathcal{F}}^{2}+\tilde{\mathcal{G}}^{2}}-\tilde{\mathcal{F}}\right)^{1 / 2}
$$

The analogous expression for $\tilde{\mathcal{F}}<0$ follows from eq. (B.9) by the transformation $\tilde{\eta} \leftrightarrow-i \tilde{\epsilon}$. Note that for $\tilde{\mathcal{F}} \gg 1$, we have

$$
\tilde{\eta}=\sqrt{2 \tilde{\mathcal{F}}}\left(1+\mathcal{O}\left(\left(\frac{\tilde{\mathcal{G}}^{2}}{\tilde{\mathcal{F}}^{2}}\right)^{2}\right)\right) \quad \text { and } \quad \tilde{\epsilon}=\frac{|\tilde{\mathcal{G}}|}{\sqrt{2 \tilde{\mathcal{F}}}}\left(1+\mathcal{O}\left(\left(\frac{\tilde{\mathcal{G}}^{2}}{\tilde{\mathcal{F}}^{2}}\right)^{2}\right)\right) .
$$

Aiming at determining the leading strong-field asymptotics of $\left.\Pi_{2 \text {-loops }}^{\mu \nu}\left(p, p^{\prime}\right)\right|_{\text {const. }}$, we need the complete scaling of the leading contribution $\sim \tilde{\mathcal{G}}^{2}$, for which the terms given in eq. (B.9) are not sufficient.

For this, we have to consider the exact double integral representation of $\left.\mathcal{L}_{\mathrm{HE}}^{2 \text {-loop }}\right|_{1 \mathrm{PI}}$ in constant external fields [65]. In the notation of [65], but adopting the sequential substitutions $s^{\prime} \rightarrow s \nu$, e $\eta s \rightarrow-i \tau$, introducing the dimensionless parameters $\tilde{\eta}=\frac{e \eta}{m^{2}}, \tilde{\epsilon}=\frac{e \epsilon}{m^{2}}$ and defining $\kappa \equiv \frac{\tilde{\epsilon}}{\tilde{\eta}},\left.\mathcal{L}_{\mathrm{HE}}^{2-\text {-loop }}\right|_{1 \mathrm{PI}}$ is given by

$$
\frac{\left.\mathcal{L}_{\mathrm{HE}}^{2 \text {-loop }}\right|_{1 \mathrm{PI}}}{m^{4}}=\frac{\alpha}{\pi} \frac{\tilde{\eta}^{2}}{16 \pi^{2}} \int_{0}^{\infty} \mathrm{d} \tau \int_{0}^{1} \mathrm{~d} \nu\left\{K(\tau, \nu)-\frac{K_{0}(\tau)}{\nu}+K_{0}(\tau)\left[\ln \left(\frac{\tau}{\tilde{\eta}}\right)+\gamma-\frac{5}{6}\right]\right\}
$$

with

$$
K_{0}(\tau)=e^{-\frac{\tau}{\tilde{\eta}}}\left(\frac{4}{\tilde{\eta}}-\partial_{\tau}\right)\left[\frac{\kappa}{\tanh (\tau) \tan (\kappa \tau)}-\frac{1}{\tau^{2}}-\frac{1-\kappa^{2}}{3}\right]
$$

and

$$
\begin{aligned}
K(\tau, \nu)= & e^{-\frac{\tau}{\tilde{\eta}}(1+\nu)}\left\{\frac{\kappa^{2}}{P P^{\prime}}\left[\frac{4}{\tilde{\eta}}\left(S S^{\prime}+P P^{\prime}\right) I_{0}+2 I\right] \tau\right. \\
& \left.-\frac{1}{\nu(1+\nu) \tau^{3}}\left[\frac{4}{\tilde{\eta}} \tau+\frac{2}{1+\nu}-\frac{1-\kappa^{2}}{3} \tau^{2}\left(\frac{2}{\tilde{\eta}}\left(\nu-2-2 \nu^{2}\right) \tau+\frac{5 \nu}{1+\nu}\right)\right]\right\} .
\end{aligned}
$$

Here, we have used

$$
\begin{aligned}
S(\tau) & =\cosh (\tau) \cos (\kappa \tau), & P(\tau) & =\sinh (\tau) \sin (\kappa \tau), \\
S^{\prime} & =S(\nu \tau), & P^{\prime} & =P(\nu \tau), \\
I_{0} & =\frac{1}{(b-a)} \ln \left(\frac{b}{a}\right), & I & =\frac{(q-p)}{(b-a)^{2}} \ln \left(\frac{b}{a}\right)-\frac{1}{(b-a)}\left(\frac{q}{b}-\frac{p}{a}\right), \\
a & =\operatorname{coth}(\tau)+\operatorname{coth}(\nu \tau), & b & =\kappa[\cot (\kappa \tau)+\cot (\kappa \nu \tau)], \\
p & =\frac{\cos [\kappa(1-\nu) \tau]}{\sinh (\tau) \sinh (\nu \tau)}, & q & =\frac{\kappa^{2} \cosh [(1-\nu) \tau]}{\sin (\kappa \tau) \sin (\kappa \nu \tau)} .
\end{aligned}
$$

Without loss of generality, we subsequently focus on the limit of $\frac{1}{\tilde{\eta}} \rightarrow 0$; the opposite limit of $\frac{1}{\tilde{\epsilon}} \rightarrow 0$ can be easily obtained by the transformation $\tilde{\eta} \leftrightarrow-i \tilde{\epsilon}$. Due to the overall exponential suppression of both $K_{0}$ and $K$ with $e^{-\frac{\tau}{\bar{\eta}}}$, the dominant contributions to 
$\left.\mathcal{L}_{\mathrm{HE}}^{2 \text {-loop }}\right|_{1 \mathrm{PI}}$ in the limit of $\frac{1}{\tilde{\eta}} \rightarrow 0$ stem from large values of $\tau$. In a first step we infer that

$$
K_{0}(\tau)=e^{-\frac{\tau}{\tilde{\eta}}}\left\{\partial_{\tau}\left[\frac{1}{\tau^{2}}-\frac{\kappa}{\tanh (\tau) \tan (\kappa \tau)}+\frac{1-\kappa^{2}}{3}\right]+\mathcal{O}\left(\left(\frac{1}{\tilde{\eta}}\right)^{1}\right)\right\}
$$

and

$$
K(\tau, \nu)=e^{-\frac{\tau}{\tilde{\eta}}(1+\nu)}\left\{\frac{\kappa^{2}}{P P^{\prime}} 2 I \tau-\frac{1}{(1+\nu)^{2}}\left[\frac{2}{\nu} \frac{1}{\tau^{2}}-5 \frac{1-\kappa^{2}}{3}\right] \frac{1}{\tau}+\mathcal{O}\left(\left(\frac{1}{\tilde{\eta}}\right)^{1}\right)\right\} .
$$

We have explicitly checked that the terms denoted by $\mathcal{O}\left(\left(\frac{1}{\tilde{n}}\right)^{1}\right)$ in eqs. (B.15) and (B.16) do not increase with $\tau$ for $\tau \rightarrow \infty$, but scale at least as $\mathcal{O}\left(\left(\frac{1}{\tau}\right)^{0}\right)$ at any given order in an expansion in $\kappa \rightarrow 0$. Moreover, note that at any fixed order in $\kappa \rightarrow 0$, we have $\lim _{\tau \rightarrow \infty}\left|\frac{I}{P P^{\prime}} \tau\right| \sim \tau^{l} e^{-\tau(1+2 \nu)} \rightarrow 0$, with $l \in \mathbb{Z}_{0}$. Herewith, we obtain

$$
\int_{0}^{\infty} \mathrm{d} \tau K_{0}(\tau)=\mathcal{O}\left(\left(\frac{1}{\tilde{\eta}}\right)^{0}\right) \mathcal{O}\left(\kappa^{0}\right)
$$

and

$$
\int_{0}^{\infty} \mathrm{d} \tau K(\tau, \nu)=\frac{5}{(1+\nu)^{2}} \frac{1-\kappa^{2}}{3} \ln \tilde{\eta}+\mathcal{O}\left(\left(\frac{1}{\tilde{\eta}}\right)^{0}\right) \mathcal{O}\left(\kappa^{0}\right)
$$

Moreover, we are interested in the following integral:

$$
\begin{aligned}
\int_{0}^{\infty} \mathrm{d} \tau K_{0}(\tau)\left[\ln \left(\frac{\tau}{\tilde{\eta}}\right)+\gamma-\frac{5}{6}\right]=\int_{0}^{\infty} \frac{\mathrm{d} \tau}{\tau} e^{-\frac{\tau}{\tilde{\eta}}}\left[\frac{\operatorname{coth}(\tau)}{\tau}-\frac{1}{\tau^{2}}-\frac{1}{3}\right] \\
+\frac{\kappa^{2}}{3} \int_{0}^{\infty} \mathrm{d} \tau e^{-\frac{\tau}{\tilde{\eta}}}\left[\frac{1}{\tau}-\operatorname{coth}(\tau)\right]+\mathcal{O}\left(\left(\frac{1}{\tilde{\eta}}\right)^{0}\right) \mathcal{O}\left(\kappa^{0}\right)+\mathcal{O}\left(\kappa^{4}\right) .
\end{aligned}
$$

In order to arrive at this result we have made use of eq. (B.15) for $K_{0}(\tau)$ and performed an integration by parts. Thereafter, we have employed an expansion in $\kappa \rightarrow 0$, keeping terms up to order $\kappa^{2}$ only. The integrals in eq. (B.19) can be carried out with formulae 3.381.4 and 3.551.3 of [91]: $\int_{0}^{\infty} \frac{\mathrm{d} \tau}{\tau} \tau^{\nu} e^{-\beta \tau}=\beta^{-\nu} \Gamma(\nu)$ and $\int_{0}^{\infty} \frac{\mathrm{d} \tau}{\tau} \tau^{\nu} e^{-\beta \tau} \operatorname{coth}(\tau)=\left[2^{1-\nu} \zeta\left(\nu, \frac{\beta}{2}\right)-\right.$ $\left.\beta^{-\nu}\right] \Gamma(\nu)$, valid for $\Re(\beta)>0$ and under certain conditions on $\nu$, which are rendered irrelevant upon combination of these formulae in performing the manifestly finite integrals in eq. (B.19). Such integral expressions are common in strong-field QED; cf., e.g., [4, 5, $68,88,92,93]$. We infer

$$
\int_{0}^{\infty} \mathrm{d} \tau e^{-\beta \tau}\left[\frac{1}{\tau}-\operatorname{coth}(\tau)\right]=\psi\left(\frac{\beta}{2}\right)+\frac{1}{\beta}-\ln \frac{\beta}{2}=-\frac{1}{\beta}-\ln \beta+\mathcal{O}\left(\beta^{0}\right),
$$

where $\psi(\chi)$ is the Digamma function (cf. section B.1), and

$$
\begin{aligned}
\int_{0}^{\infty} \frac{\mathrm{d} \tau}{\tau} e^{-\beta \tau}\left[\frac{\operatorname{coth}(\tau)}{\tau}-\frac{1}{\tau^{2}}-\frac{1}{3}\right] & =\frac{1}{3}\left(\ln \frac{\beta}{2}+1\right)-\beta \ln \frac{\beta}{2}-4 \zeta^{\prime}\left(-1, \frac{\beta}{2}\right)+\frac{\beta^{2}}{2}\left(\ln \frac{\beta}{2}-\frac{1}{2}\right) \\
& =\frac{1}{3} \ln \beta+\mathcal{O}\left(\beta^{0}\right),
\end{aligned}
$$


where $\zeta^{\prime}(-1, \chi)$ is the first derivative of the Hurwitz zeta function (B.5). Using eqs. (B.20) and (B.21) in eq. (B.19), we finally obtain

$$
\int_{0}^{\infty} \mathrm{d} \tau K_{0}(\tau)\left[\ln \left(\frac{\tau}{\tilde{\eta}}\right)+\gamma-\frac{5}{6}\right]=-\frac{1}{3}\left[\left(1-\kappa^{2}\right) \ln \tilde{\eta}+\kappa^{2} \tilde{\eta}\right]+\mathcal{O}\left(\left(\frac{1}{\tilde{\eta}}\right)^{0}\right) \mathcal{O}\left(\kappa^{0}\right)+\mathcal{O}\left(\kappa^{4}\right) .
$$

Putting everything together, we hence have

$$
\frac{\left.\mathcal{L}_{\mathrm{HE}}^{2 \text {-loop }}\right|_{1 \mathrm{PI}}}{m^{4}}=\frac{\alpha}{\pi} \frac{\tilde{\eta}^{2}}{32 \pi^{2}}\left\{\left(1-\kappa^{2}\right) \ln \tilde{\eta}-\frac{2}{3} \kappa^{2} \tilde{\eta}+\mathcal{O}\left(\left(\frac{1}{\tilde{\eta}}\right)^{0}\right) \mathcal{O}\left(\kappa^{0}\right)+\mathcal{O}\left(\kappa^{4}\right)\right\} .
$$

In a last step, we employ [cf. eq. (B.10)]

$$
\kappa^{2}=\frac{\tilde{\mathcal{G}}^{2}}{(2 \tilde{\mathcal{F}})^{2}}+\mathcal{O}\left(\left(\frac{\tilde{\mathcal{G}}^{2}}{\tilde{\mathcal{F}}^{2}}\right)^{2}\right) \quad \text { and } \quad \tilde{\eta}=\sqrt{2 \tilde{\mathcal{F}}}\left(1+\mathcal{O}\left(\left(\frac{\tilde{\mathcal{G}}^{2}}{\tilde{\mathcal{F}}^{2}}\right)^{1}\right)\right)
$$

to write eq. (B.23) in the form of eq. (3.7) in the main text.

Open Access. This article is distributed under the terms of the Creative Commons Attribution License (CC-BY 4.0), which permits any use, distribution and reproduction in any medium, provided the original author(s) and source are credited.

\section{References}

[1] H. Euler and B. Kockel, Über die Streuung von Licht an Licht nach der Diracschen Theorie, Naturwiss. 23 (1935) 246 [INSPIRE].

[2] W. Heisenberg and H. Euler, Folgerungen aus der Diracschen Theorie des Positrons, Z. Phys. 98 (1936) 714 [physics/0605038] [INSPIRE].

[3] V. Weisskopf, Über die Elektrodynamik des Vakuums auf Grund der Quanthentheorie des Elektrons, Kong. Dans. Vid. Selsk. Mat.-fys. Medd. XIV (1936) 6.

[4] W. Dittrich and M. Reuter, Effective lagrangians in quantum electrodynamics, Lect. Notes Phys. 220 (1985) 1 [INSPIRE].

[5] W. Dittrich and H. Gies, Probing the quantum vacuum. Perturbative effective action approach in quantum electrodynamics and its application, Springer Tracts Modern Physicis volume 166, Springer, Germany (2000).

[6] M. Marklund and J. Lundin, Quantum vacuum experiments using high intensity lasers, Eur. Phys. J. D 55 (2009) 319 [arXiv:0812.3087] [InSPIRE].

[7] G.V. Dunne, New strong-field QED effects at ELI: nonperturbative vacuum pair production, Eur. Phys. J. D 55 (2009) 327 [arXiv:0812.3163] [InSPIRE].

[8] T. Heinzl and A. Ilderton, Exploring high-intensity QED at ELI, Eur. Phys. J. D 55 (2009) 359 [arXiv: 0811.1960] [INSPIRE].

[9] A. Di Piazza, C. Muller, K.Z. Hatsagortsyan and C.H. Keitel, Extremely high-intensity laser interactions with fundamental quantum systems, Rev. Mod. Phys. 84 (2012) 1177 [arXiv:1111.3886] [INSPIRE].

[10] G.V. Dunne, The Heisenberg-Euler effective action: 75 years on, Int. J. Mod. Phys. A 27 (2012) 1260004 [Int. J. Mod. Phys. Conf. Ser. 14 (2012) 42] [arXiv:1202.1557] [inSPIRE]. 
[11] R. Battesti and C. Rizzo, Magnetic and electric properties of quantum vacuum, Rept. Prog. Phys. 76 (2013) 016401 [arXiv: 1211.1933] [INSPIRE].

[12] B. King and T. Heinzl, Measuring vacuum polarisation with high power lasers, High Power Laser Science and Engineering 4, Cambridge University Press, Cambridge U.K. (2016), arXiv:1510.08456 [INSPIRE].

[13] F. Karbstein, The quantum vacuum in electromagnetic fields: from the Heisenberg-Euler effective action to vacuum birefringence, arXiv:1611.09883 [INSPIRE].

[14] F. Sauter, Über das Verhalten eines Elektrons im homogenen elektrischen Feld nach der relativistischen Theorie Diracs, Z. Phys. 69 (1931) 742 [INSPIRE].

[15] J.S. Schwinger, On gauge invariance and vacuum polarization, Phys. Rev. 82 (1951) 664 [INSPIRE].

[16] J.S. Toll, The dispersion relation for light and its application to problems involving electron pairs, Ph.D. thesis, Princeton University, Princeton, U.S.A. (1952).

[17] R. Baier and P. Breitenlohner, Photon Propagation in external fields, Act. Phys. Austriaca 25 (1967) 212.

[18] R. Baier and P. Breitenlohner, The vacuum refraction index in the presence of external fields, Nuov. Cim. B 47 (1967) 117.

[19] Z. Bialynicka-Birula and I. Bialynicki-Birula, Nonlinear effects in quantum electrodynamics. Photon propagation and photon splitting in an external field, Phys. Rev. D 2 (1970) 2341 [INSPIRE].

[20] PVLAS collaboration, G. Cantatore, Recent results from the PVLAS experiment on the magnetized vacuum, Lect. Notes Phys. 741 (2008) 157 [INSPIRE].

[21] PVLAS collaboration, E. Zavattini et al., New PVLAS results and limits on magnetically induced optical rotation and ellipticity in vacuum, Phys. Rev. D 77 (2008) 032006 [arXiv:0706.3419] [INSPIRE].

[22] F. Della Valle et al., Measurements of vacuum magnetic birefringence using permanent dipole magnets: the PVLAS experiment, New J. Phys. 15 (2013) 053026 [arXiv:1301.4918] [INSPIRE].

[23] P. Berceau, R. Battesti, M. Fouche and C. Rizzo, The vacuum magnetic birefringence experiment: A test for quantum electrodynamics, Can. J. Phys. 89 (2011) 153.

[24] P. Berceau et al., Magnetic linear birefringence measurements using pulsed fields, Phys. Rev. A 85 (2012) 013837 [arXiv: 1109.4792].

[25] A. Cadène, P. Berceau, M. Fouché, R. Battesti and C. Rizzo, Vacuum magnetic linear birefringence using pulsed fields: status of the BMV experiment, Eur. Phys. J. D 68 (2014) 16 [arXiv: 1302.5389] [INSPIRE].

[26] G.L. Kotkin and V.G. Serbo, Variation in polarization of high-energy gamma quanta traversing a bunch of polarized laser photons, Phys. Lett. B 413 (1997) 122 [hep-ph/9611345] [INSPIRE].

[27] T. Heinzl, B. Liesfeld, K.-U. Amthor, H. Schwoerer, R. Sauerbrey and A. Wipf, On the observation of vacuum birefringence, Opt. Commun. 267 (2006) 318 [hep-ph/0601076] [INSPIRE]. 
[28] A. Di Piazza, K.Z. Hatsagortsyan and C.H. Keitel, Light diffraction by a strong standing electromagnetic wave, Phys. Rev. Lett. 97 (2006) 083603 [hep-ph/0602039] [INSPIRE].

[29] V. Dinu, T. Heinzl, A. Ilderton, M. Marklund and G. Torgrimsson, Vacuum refractive indices and helicity flip in strong-field QED, Phys. Rev. D 89 (2014) 125003 [arXiv:1312.6419] [INSPIRE].

[30] V. Dinu, T. Heinzl, A. Ilderton, M. Marklund and G. Torgrimsson, Photon polarization in light-by-light scattering: Finite size effects, Phys. Rev. D 90 (2014) 045025 [arXiv: 1405.7291] [INSPIRE].

[31] A. Ilderton and M. Marklund, Prospects for studying vacuum polarisation using dipole and synchrotron radiation, J. Plasma Phys. 82 (2016) 655820201 [arXiv: 1601.08045] [INSPIRE].

[32] B. King and N. Elkina, Vacuum birefringence in high-energy laser-electron collisions, Phys. Rev. A 94 (2016) 062102 [arXiv: 1603.06946] [INSPIRE].

[33] H.P. Schlenvoigt et al., Detecting vacuum birefringence with x-ray free electron lasers and high-power optical lasers: a feasibility study, Phys. Scripta 91 (2016) 023010.

[34] F. Karbstein, H. Gies, M. Reuter and M. Zepf, Vacuum birefringence in strong inhomogeneous electromagnetic fields, Phys. Rev. D 92 (2015) 071301 [arXiv:1507.01084] [INSPIRE].

[35] F. Karbstein and C. Sundqvist, Probing vacuum birefringence using $x$-ray free electron and optical high-intensity lasers, Phys. Rev. D 94 (2016) 013004 [arXiv: 1605.09294] [INSPIRE].

[36] R.P. Mignani et al., Evidence for vacuum birefringence from the first optical polarimetry measurement of the isolated neutron star RX J1856.5-3754, Mon. Not. R. Astron. Soc. 465 (2017) 492 [arXiv : 1610.08323] [INSPIRE].

[37] E. Lundstrom et al., Using high-power lasers for detection of elastic photon-photon scattering, Phys. Rev. Lett. 96 (2006) 083602 [hep-ph/0510076] [INSPIRE].

[38] J. Lundin et al., Detection of elastic photon-photon scattering through four-wave mixing using high power lasers, Phys. Rev. A 74 (2006) 043821 [hep-ph/0606136] [INSPIRE].

[39] D. Tommasini and H. Michinel, Light by light diffraction in vacuum, Phys. Rev. A 82 (2010) 011803 [arXiv: 1003. 5932] [INSPIRE].

[40] B. King and C.H. Keitel, Photon-photon scattering in collisions of laser pulses, New J. Phys. 14 (2012) 103002 [arXiv:1202.3339] [INSPIRE].

[41] B. King, A. Di Piazza and C.H. Keitel, A matterless double slit, Nature Photon. 4 (2010) 92 [arXiv: 1301.7038] [INSPIRE].

[42] B. King, A. Di Piazza and C.H. Keitel, Double-slit vacuum polarisation effects in ultra-intense laser fields, Phys. Rev. A 82 (2010) 032114 [arXiv:1301.7008] [INSPIRE].

[43] K.Z. Hatsagortsyan and G.Y. Kryuchkyan, Bragg scattering of light in vacuum structured by strong periodic fields, Phys. Rev. Lett. 107 (2011) 053604.

[44] X. Sarazin et al., Refraction of light by light in vacuum, Eur. Phys. J. D 70 (2016) 13 [INSPIRE].

[45] H. Gies, F. Karbstein and N. Seegert, Quantum reflection as a new signature of quantum vacuum nonlinearity, New J. Phys. 15 (2013) 083002 [arXiv:1305.2320] [INSPIRE]. 
[46] H. Gies, F. Karbstein and N. Seegert, Quantum reflection of photons off spatio-temporal electromagnetic field inhomogeneities, New J. Phys. 17 (2015) 043060 [arXiv:1412.0951] [INSPIRE].

[47] V.P. Yakovlev, Incoherent electromagnetic wave scattering in a Coulomb field,Sov. Phys. JETP 24 (1967) 411 [Zh. Eksp. Teor. Fiz. 51 (1966) 619].

[48] A. Di Piazza, K.Z. Hatsagortsyan and C.H. Keitel, Non-perturbative vacuum-polarization effects in proton-laser collisions, Phys. Rev. Lett. 100 (2008) 010403 [arXiv:0708.0475] [INSPIRE].

[49] A. Di Piazza, K.Z. Hatsagortsyan and C.H. Keitel, Laser photon merging in proton-laser collisions, Phys. Rev. A 78 (2008) 062109 [arXiv:0906.5576] [INSPIRE].

[50] H. Gies, F. Karbstein and R. Shaisultanov, Laser photon merging in an electromagnetic field inhomogeneity, Phys. Rev. D 90 (2014) 033007 [arXiv:1406.2972] [INSPIRE].

[51] H. Gies, F. Karbstein and N. Seegert, Photon merging and splitting in electromagnetic field inhomogeneities, Phys. Rev. D 93 (2016) 085034 [arXiv:1603.00314] [INSPIRE].

[52] S.L. Adler, Photon splitting and photon dispersion in a strong magnetic field, Annals Phys. 67 (1971) 599 [INSPIRE].

[53] S.L. Adler, J.N. Bahcall, C.G. Callan and M.N. Rosenbluth, Photon splitting in a strong magnetic field, Phys. Rev. Lett. 25 (1970) 1061 [INSPIRE].

[54] V.O. Papanyan and V.I. Ritus, Vacuum polarization and photon splitting in an intense field, Sov. Phys. JETP 34 (1972) 1195 [Zh. Eksp. Teor. Fiz. 61 (1971) 2231].

[55] V.O. Papanyan and V.I. Ritus, Three-photon interaction in an intense field and scaling invariance, Sov. Phys. JETP 38 (1974) 879 [Zh. Eksp. Teor. Fiz. 65 (1973) 1756] [INSPIRE].

[56] R.J. Stoneham, Phonon splitting in the magnetised vacuum, J. Phys. A 12 (1979) 2187.

[57] V.N. Baier, A.I. Milshtein and R.Z. Shaisultanov, Photon splitting in a strong electromagnetic field, Sov. Phys. JETP 63 (1986) 665 [Zh. Eksp. Teor. Fiz. 90 (1986) 1141] [inSPIRE].

[58] V.N. Baier, A.I. Milshtein and R.Z. Shaisultanov, Photon splitting in a very strong magnetic field, Phys. Rev. Lett. 77 (1996) 1691 [hep-th/9604028] [InSPIRE].

[59] S.L. Adler and C. Schubert, Photon splitting in a strong magnetic field: Recalculation and comparison with previous calculations, Phys. Rev. Lett. 77 (1996) 1695 [hep-th/9605035] [INSPIRE].

[60] A. Di Piazza, A.I. Milstein and C.H. Keitel, Photon splitting in a laser field, Phys. Rev. A 76 (2007) 032103 [arXiv: 0704.0695] [INSPIRE].

[61] A. Di Piazza, K.Z. Hatsagortsyan and C.H. Keitel, Harmonic generation from laser-driven vacuum, Phys. Rev. D 72 (2005) 085005 [INSPIRE].

[62] A.M. Fedotov and N.B. Narozhny, Generation of harmonics by a focused laser beam in vacuum, Phys. Lett. A 362 (2007) 1 [hep-ph/0604258] [INSPIRE].

[63] F. Karbstein and R. Shaisultanov, Stimulated photon emission from the vacuum, Phys. Rev. D 91 (2015) 113002 [arXiv:1412.6050] [INSPIRE].

[64] P. Böhl, B. King and H. Ruhl, Vacuum high harmonic generation in the shock regime, Phys. Rev. A 92 (2015) 032115 [arXiv:1503.05192] [InSPIRE]. 
[65] V.I. Ritus, The lagrange function of an intensive electromagnetic field and quantum electrodynamics at small distances, Sov. Phys. JETP 42 (1975) 774 [Zh. Eksp. Teor. Fiz. 69 (1975) 1517] [INSPIRE].

[66] W. Dittrich and H. Gies, Light propagation in nontrivial QED vacua, Phys. Rev. D 58 (1998) 025004 [hep-ph/9804375] [INSPIRE].

[67] V.I. Ritus, Connection between strong-field quantum electrodynamics with shortdistance quantum electrodynamics, Sov. Phys. JETP 46 (1977) 423 [Zh. Eksp. Teor. Fiz. 73 (1977) 807].

[68] F. Karbstein and R. Shaisultanov, Photon propagation in slowly varying inhomogeneous electromagnetic fields, Phys. Rev. D 91 (2015) 085027 [arXiv:1503.00532] [INSPIRE].

[69] D. Fliegner, M. Reuter, M.G. Schmidt and C. Schubert, The Two loop Euler-Heisenberg Lagrangian in dimensional renormalization, Theor. Math. Phys. 113 (1997) 1442 [Teor. Mat. Fiz. 113 (1997) 289] [hep-th/9704194] [INSPIRE].

[70] B. Körs and M.G. Schmidt, The Effective two loop Euler-Heisenberg action for scalar and spinor QED in a general constant background field, Eur. Phys. J. C 6 (1999) 175 [hep-th/9803144] [INSPIRE].

[71] G.V. Dunne, Heisenberg-Euler effective lagrangians: basics and extensions, in From fields to string. Volume 1, M. Shifman ed., World Scientific, Singapore (2005), hep-th/0406216.

[72] G.V. Dunne, H. Gies and C. Schubert, Zero modes, $\beta$-functions and IR/UV interplay in higher loop QED, JHEP 11 (2002) 032 [hep-th/0210240] [INSPIRE].

[73] D. Cangemi, E. D'Hoker and G.V. Dunne, Effective energy for QED in $(2+1)$-dimensions with semilocalized magnetic fields: a solvable model, Phys. Rev. D 52 (1995) R3163 [hep-th/9506085] [INSPIRE].

[74] G.V. Dunne and T.M. Hall, An exact $(3+1)$-dimensional QED effective action, Phys. Lett. B 419 (1998) 322 [hep-th/9710062] [INSPIRE].

[75] G.V. Dunne and T. Hall, On the QED effective action in time dependent electric backgrounds, Phys. Rev. D 58 (1998) 105022 [hep-th/9807031] [INSPIRE].

[76] S.P. Kim, H.K. Lee and Y. Yoon, Effective action of QED in electric field backgrounds II. Spatially localized fields, Phys. Rev. D 82 (2010) 025015 [arXiv:0910.3363] [INSPIRE].

[77] I. Huet, D.G.C. McKeon and C. Schubert, Three-loop Euler-Heisenberg lagrangian and asymptotic analysis in $1+1$ QED, arXiv:0911.0227 [INSPIRE].

[78] I. Huet, M. Rausch de Traubenberg and C. Schubert, The Euler-Heisenberg lagrangian beyond one loop, Int. J. Mod. Phys. Conf. Ser. 14 (2012) 383 [arXiv:1112.1049] [InSPIRE].

[79] G.V. Galtsov and N.S. Nikitina, Macroscopic vacuum effects in an inhomogeneous and nonstationary electromagnetic field, Sov. Phys. JETP 57 (1983) 705 [Zh. Eksp. Teor. Fiz. 84 (1983) 1217].

[80] V.P. Gusynin and I.A. Shovkovy, Derivative expansion of the effective action for QED in $(2+1)$-dimensions and (3+1)-dimensions, J. Math. Phys. 40 (1999) 5406 [hep-th/9804143] [INSPIRE].

[81] L.C. Martin, C. Schubert and V.M. Villanueva Sandoval, On the low-energy limit of the QED N photon amplitudes, Nucl. Phys. B 668 (2003) 335 [hep-th/0301022] [inSPIRE]. 
[82] D. Galtsov and V. Skobelev, Photons creation by an external field, Phys. Lett. B 36 (1971) 238 [INSPIRE].

[83] R. Karplus and M. Neuman, Non-linear interactions between electromagnetic fields, Phys. Rev. 80 (1950) 380.

[84] R. Karplus and M. Neuman, The scattering of light by light, Phys. Rev. 83 (1951) 776 [INSPIRE].

[85] P. Bhartia and S. Valluri, Non-linear scattering of light in the limit of ultra-strong fields, Can. J. Phys. 56 (1978) 1122.

[86] S.R. Valluri and P. Bhartia, An analytical proof for the generation of higher harmonics due to the interaction of plane electromagnetic waves, Can. J. Phys. 58 (1980) 116.

[87] H. Gies, J. Jaeckel and A. Ringwald, Polarized light propagating in a magnetic field as a probe of millicharged fermions, Phys. Rev. Lett. 97 (2006) 140402 [hep-ph/0607118] [INSPIRE].

[88] W. Dittrich, One loop effective potentials in QED, J. Phys. A 9 (1976) 1171 [INSPIRE].

[89] NIST Digital Library of Mathematical Functions, http://dlmf.nist.gov/25.11\#E18, Release 1.0.10 (2015).

[90] G.V. Dunne and C. Schubert, Closed form two loop Euler-Heisenberg Lagrangian in a selfdual background, Phys. Lett. B 526 (2002) 55 [hep-th/0111134] [INSPIRE].

[91] I.S. Gradshteyn and I.M. Ryzhik, Table of integrals, series, and products, $5^{\text {th }}$ edition, Academic Press, U.S.A. (1994).

[92] W.-y. Tsai and T. Erber, The propagation of photons in homogeneous magnetic fields: index of refraction, Phys. Rev. D 12 (1975) 1132 [INSPIRE].

[93] F. Karbstein, Photon polarization tensor in a homogeneous magnetic or electric field, Phys. Rev. D 88 (2013) 085033 [arXiv: 1308.6184] [INSPIRE]. 